\title{
Uniqueness of martingale solutions for the stochastic nonlinear Schrödinger equation on 3d compact manifolds
}

\section{Zdzisław Brzeźniak ${ }^{1}$ (1) $\cdot$ Fabian Hornung ${ }^{2} \cdot$ Lutz Weis $^{2}$}

This article is dedicated to István Gyöngy on the occasion of his 70th birthday.

Received: 1 November 2021 / Revised: 1 November 2021 / Accepted: 16 December 2021 /

Published online: 2 March 2022

(c) The Author(s) 2022

\section{Abstract}

We prove the pathwise uniqueness of solutions of the nonlinear Schrödinger equation with conservative multiplicative noise on compact 3D manifolds. In particular, we generalize the result by Burq, Gérard and Tzvetkov, [7], to the stochastic setting. The proof is based on the deterministic and new stochastic spectrally localized Strichartz estimates and the Littlewood-Paley decomposition.

Keywords Nonlinear Schrödinger equation $\cdot$ Stratonovich noise $\cdot$ Strichartz estimates · Pathwise uniqueness · Littlewood-Paley decomposition

\section{Introduction and main result}

This article is concerned with the stochastic nonlinear Schrödinger equation with multiplicative noise

$$
\left\{\begin{array}{l}
\mathrm{d} u(t)=\left(\mathrm{i} \Delta_{g} u(t)-\mathrm{i} \lambda|u(t)|^{\alpha-1} u(t)\right) d t-\mathrm{i} \sum_{m=1}^{\infty} e_{m} u(t) \circ \mathrm{d} \beta_{m}(t), \quad t \in(0, T), \\
u(0)=u_{0} \in H^{1}(M),
\end{array}\right.
$$

Zdzisław Brzeźniak

zdzislaw.brzezniak@york.ac.uk

Fabian Hornung

fabianhornung89@gmail.com

Lutz Weis

lutz.weis@kit.edu

1 Department of Mathematics, University of York, Heslington, York YO105DD, UK

2 Institute for Analysis, Karlsruhe Institute for Technology (KIT), 76128 Karlsruhe, Germany 
on a compact 3D Riemannian manifold $M$, where $\Delta_{g}$ is the Laplace-Beltrami-operator, $\alpha \in(1,3], \lambda \in\{-1,1\},\left(e_{m}\right)_{m \in \mathbb{N}}$ are real valued functions and $\left(\beta_{m}\right)_{m \in \mathbb{N}}$ are independent Brownian motions. If $\lambda=1$, the NLS is called defocusing and if $\lambda=-1$, it is called focusing. In the main result of this article, we show the pathwise uniqueness of martingale solutions to Problem (1.1) in the energy space $H^{1}(M)$.

Theorem 1.1 Let $M$ be a compact $3 D$ Riemannian manifold. Let $\lambda \in\{-1,1\}, \alpha \in$ $(1,3]$ and $e_{m} \in L^{\infty}(M)$ real valued with $\nabla e_{m} \in L^{3}(M)$ for $m \in \mathbb{N}$ and

$$
\sum_{m=1}^{\infty}\left(\left\|\nabla e_{m}\right\|_{L^{3}}+\left\|e_{m}\right\|_{L^{\infty}}\right)^{2}<\infty .
$$

Then, the solutions to Problem (1.1) in the sense of Definition 2.2 are pathwise unique.

Theorem 1.1 generalizes the result by Burq, Gérard and Tzvetkov from [7], Theorem 3 , for the cubic NLS to the stochastic setting. Note that this uniqueness result holds for both the focusing and defocusing case. Combining Theorem 1.1 with the existence of martingale solutions proved by the authors in [9] and the Yamada-Watanabe-Theory developed in [36], Theorem 5.3 and Corollary 5.4, see also [41, Theorems 2 and 12.1], we obtain the existence of a unique strong solution of (1.1).

Corollary 1.2 Let $M$ be a compact $3 D$ Riemannian manifold. Let $\lambda=1$ and $\alpha \in(1,3]$ or $\lambda=-1$ and $\alpha \in\left(1, \frac{7}{3}\right)$. If $\left(e_{m}\right)_{m \in \mathbb{N}}$ satisfies the conditions from Theorem 1.1, there is a unique global strong solution to Problem (1.1), cf. Definition 2.2 below, and the martingale solutions are unique in law.

The question of the existence and the uniqueness of global solutions of the stochastic nonlinear Schrödinger equation has attracted some attention in the recent years. We refer to de Bouard and Debussche [21;22], Barbu, Röckner and Zhang [14;15;50] and the second author [30] as well as to the references in these articles. These authors considered the stochastic NLS in the Euclidean space $\mathbb{R}^{d}$ and employed a fixed point argument based on the Strichartz estimates to prove the existence and the uniqueness simultaneously. As in the deterministic setting, their range of the exponents $\alpha$ depends on the space dimension $d$ and the considered regularity. Brzeźniak and Millet followed a similar approach for the stochastic NLS on a compact 2-dimensional manifold $M$. In higher dimensions, their argument only yields the existence of local solutions since the estimates for the nonlinearity rely on the Sobolev embeddings $H^{s, p} \hookrightarrow L^{\infty}$ that are too restrictive to work in the energy space $H^{1}(M)$. Another result about the stochastic NLS is due to Keller and Lisei, see [34], who considered the equation on the one-dimensional space-interval $(0,1)$ with the Neumann boundary conditions. They proved the existence by employing the Galerkin method and proved the uniqueness via the Sobolev embedding $H^{1}(0,1) \hookrightarrow L^{\infty}(0,1)$. Hence, their argument cannot be transferred to higher dimensions. After this work was finished, we learned about a recent paper [20] by Cheung and Mosincat. Using the additional structure in the special case of the $d$-dimensional torus $M=\mathbb{T}^{d}$ and algebraic nonlinearities, i.e. $\alpha=2 k+1$ for some $k \in \mathbb{N}$, the authors employed a fixed point argument based on the multilinear Strichartz estimates and an estimate of the stochastic convolution in Bourgain spaces $X^{s, b}$ combined with the truncation 
method from $[21 ; 22 ; 30]$. As a result, they solved the stochastic NLS with multiplicative noise in the space $L^{2}\left(\Omega, C\left([0, \tau], H^{s}\left(\mathbb{T}^{d}\right)\right) \cap X^{s, b}([0, \tau])\right)$ for some stopping time $\tau>0$, for every $s>s_{\text {crit }}:=\frac{d}{2}-\frac{2}{\alpha-1}$ and some $b<\frac{1}{2}$. As a byproduct, their argument also implies the pathwise uniqueness of the martingale solutions in $L^{2}\left(\Omega, C\left([0, T], H^{s}\left(\mathbb{T}^{3}\right)\right) \cap X^{s, b}([0, T])\right)$ for $\alpha=3$ and $s>\frac{1}{2}$, which reflects an improvement on the torus compared to the general case considered in our Theorem 1.1.

Let us now explain our approach to the existence and the uniqueness and the main contributions of the present paper. Instead of using a fixed point argument, we separate the proof of the existence and the uniqueness. The construction of a martingale solution has been treated by the authors in [9] and the second author in [31] based on the Hamiltonian structure of the NLS without using the Strichartz estimates. Since these ingredients are independent of the underlying geometry, the existence proof works in a more general framework including non-compact manifolds and domains with Neumann or Dirichlet boundary in arbitrary dimension. The flexibility of this approach is underlined by the fact that it could be also used to construct a martingale solution of the NLS with pure jump noise, see [8]. In a second step, we consider the pathwise uniqueness of the martingale solution with additional assumptions on the noise and the geometry in order to profit from the dispersive properties of the Schrödinger equation. In this way, we have proved an analogue of Theorem 1.1 in two dimensions in [9].

In the present article, we turn our attention to the case of three dimensions. To the best knowledge of the authors our paper is the first one in which the uniqueness of solutions to the stochastic NLS on a 3-dimensional compact Riemannian manifold driven by linear multiplicative noise has been established. The main technical ingredient in the proof of Theorem 1.1 is the estimate

$$
\|u\|_{L^{2}\left(J, L^{p}\right)} \lesssim 1+(|J| p)^{\frac{1}{2}} \quad \text { a.s. }
$$

of any martingale solution to Problem (1.1) in terms of the length of time interval $J$ and the exponent $p \in[6, \infty)$. We refer to Proposition 3.1 for an exact formulation of this estimate. It is obtained by employing the Littlewood-Paley decomposition and a partition of unity of the time interval followed by the spectrally localized Strichartz estimates on short time intervals, cf. for instance inequality (1.5) below. Hereby, the main novelty of our approach is the spectrally localized Strichartz estimate for stochastic convolution with the Schrödinger group established in Lemma 2.12 and its usage to get a pathwise estimate of the stochastic convolution which can handle the stochastic term in Eq. (1.1). Note that the estimate (1.3) is sharp in the sense that it cannot be proved for $\alpha>3$. In the cubic case $\alpha=3$, the endpoint Strichartz inequality of Keel and Tao [35] is required to estimate the most critical terms.

We now sketch the proof of Theorem 1.1 and thereby explain the role of the estimate (1.3) to overcome the additional difficulties posed by the 3-dimensional setting compared to the 2-dimensional one. We take two solutions with $u_{1}, u_{2} \in$ $L^{\infty}\left(0, T ; H^{1}(M)\right)$ almost surely. Our starting point is the representation

$$
\begin{aligned}
\left\|u_{1}(t)-u_{2}(t)\right\|_{L^{2}}^{2}= & 2 \int_{0}^{t} \operatorname{Re}\left(u_{1}(s)-u_{2}(s),-\mathrm{i} \lambda\left|u_{1}(s)\right|^{\alpha-1} u_{1}(s)\right. \\
& \left.+\mathrm{i}\left|u_{2}(s)\right|^{\alpha-1} u_{2}(s)\right)_{L^{2}} \mathrm{~d} s
\end{aligned}
$$


which holds almost surely for all $t \in[0, T]$. To get this identity, it is crucial to consider Eq. (1.1) with the noise in the Stratonovich form with real valued coefficients, since this leads to cancellations of the stochastic integral and the correction term in the Itô formula. We remark that the formula (1.4) is closely related to the mass conservation of solutions to (1.1) which leads to the notion of conservative noise. To use equality (1.4) for the uniqueness proof, one can employ the following local Strichartz estimate

$$
\left\|t \mapsto e^{\mathrm{i} t \Delta_{g}} \varphi\left(h^{2} \Delta_{g}\right) x\right\|_{L^{q}\left(0, T ; L^{p}\right)} \lesssim\|x\|_{L^{2}}, \quad x \in L^{2}(M),
$$

for small times $T \lesssim h$ and the global Strichartz estimate

$$
\left\|t \mapsto e^{\mathrm{i} t \Delta_{g}} x\right\|_{L^{q}\left(0, T ; L^{p}(M)\right)} \lesssim\|x\|_{H^{\frac{1}{q}}(M)}, \quad x \in H^{\frac{1}{q}}(M) .
$$

from [7] for every Strichartz-admissible exponent pairs $(p, q) \in[2, \infty]^{2}$. Here, $h \in$ $(0,1]$ and $\varphi \in C_{c}^{\infty}(\mathbb{R})$ can be chosen arbitrarily.

In the 2-dimensional case $d=2$, inequality (1.6) allows one to deduce an improved regularity $L^{q}\left(0, T ; H^{s-\frac{1}{q}, p}\right)$, for $s \in\left(1-\frac{1}{q}, 1\right)$, almost surely, of the processes $u_{1}, u_{2}$. Hence, one can use a Gronwall Lemma type argument based on the Sobolev embedding $H^{s-\frac{1}{q}, p}(M) \hookrightarrow L^{\infty}(M)$ to prove the pathwise uniqueness. For the details, we refer to [9]. In $3 \mathrm{D}$, the challenge is to gain $\frac{1}{2}+\varepsilon$ derivatives with respect to the embedding $H^{\frac{3}{2}+\varepsilon}(M) \hookrightarrow L^{\infty}(M)$ in order to control the nonlinearity in (1.4) by the $H^{1}$-estimates of the solutions. Unfortunately, this is not possible, but it turns out that (1.3) is a good substitute for the missing $L^{\infty}$-estimate.

To deduce the pathwise uniqueness from (1.3), we generalize a strategy developed by Yudovich, [49], for the Euler equation to the stochastic setting. In the context of the deterministic NLS, it is also well-known and has been used already by Vladimirov in [48], Ogawa and Ozawa in [40] and [42]. They considered the 2-dimensional domains and used the Trudinger type inequalities as an analogue of inequality (1.3) to control the growth of $L^{p}$-norms for $p \rightarrow \infty$. Burq, Gérard and Tzvetkov could use the Yudovichstrategy for three dimensional manifolds without boundary due to the regularizing effect of the Strichartz estimates. In [17], Blair, Smith and Sogge proved the uniqueness of weak solutions of the deterministic NLS on compact $3 D$ manifolds with boundary as an application of their Strichartz estimates on this type of geometry.

The paper is organized as follows. In Sect. 2, we fix the notations, formulate our assumptions and collect auxiliary results. Section 3 is devoted to proof of the estimate (1.3) and the pathwise uniqueness.

\section{Definitions and auxiliary results}

This section is devoted to the notations, definitions and auxiliary results that will be used in the next section to show the pathwise uniqueness.

If $a, b \geq 0$ satisfy the inequality $a \leq C b$ with a constant $C>0$, we write $a \lesssim b$. Given $a \lesssim b$ and $b \lesssim a$, we write $a \approx b$. For two Banach spaces $E, F$, we denote by $\mathcal{L}(E, F)$ the space of linear bounded operators $B: E \rightarrow F$ and abbreviate $\mathcal{L}(E):=\mathcal{L}(E, E)$. We use the notation $\operatorname{HS}\left(H_{1}, H_{2}\right)$ for the space of Hilbert-Schmidt- 
operators between Hilbert spaces $H_{1}$ and $H_{2}$. Furthermore, we write $E \hookrightarrow F$ if $E$ is continuously embedded in $F$; i.e. $E \subset F$ with natural embedding $j \in \mathcal{L}(E, F)$.

Let $M$ be a three dimensional compact Riemannian $C^{\infty}$ manifold without boundary and $L^{p}(M)$ for $p \in[1, \infty]$ the space equivalence classes of $\mathbb{C}$-valued $p$-integrable functions. The distance induced by $g$ is denoted by $\rho$ and canonical measure on $M$ is called $\mu$. By $L^{p}(M)$ for $p \in[1, \infty]$, we denote the space of equivalence classes of $\mathbb{C}$-valued $p$-integrable functions with respect to $\mu$. The Laplace-Beltrami operator on $M$, i.e. the generator of the heat semigroup on $M$, is named $\Delta_{g}$. Moreover, we use the fractional Sobolev spaces

$$
H^{s, p}(M):=\left\{u \in L^{p}(M): \exists v \in L^{p}(M): u=\left(I-\Delta_{g}\right)^{-\frac{s}{2}} v\right\}
$$

for $p \in[1, \infty)$ and $s \geq 0$ with the norm $\|u\|_{H^{s, p}}:=\|v\|_{L^{p}}$. For $s<0$, the space $H^{s, p}(M)$ is defined as the completion of $L^{p}(M)$ with respect to

$$
\|u\|_{H^{s, p}}:=\left\|\left(I-\Delta_{g}\right)^{\frac{s}{2}} u\right\|_{L^{p}}, \quad u \in L^{p}(M) .
$$

For all $s \in \mathbb{R}$, we shortly denote $H^{s}(M):=H^{s, 2}(M)$. For properties of the LaplaceBeltrami operator, characterizations of the fractional Sobolev spaces and embedding theorems, we refer to [46] and [44]. For $s=1$, one can show that the definition from above coincides with the classical Sobolev space and $\left(\|u\|_{L^{2}}^{2}+\|\nabla u\|_{L^{2}}^{2}\right)^{\frac{1}{2}}$ defines an equivalent norm on $H^{1}(M)$. We refer to [38] for an explanation of the gradient as an element of the tangential bundle of $M$.

Next, we summarize the assumptions on the coefficient of the noise in Problem (1.1).

Assumption 2.1 Let $Y$ be a separable Hilbert space and $B: H^{1}(M) \rightarrow \operatorname{HS}\left(Y, H^{1}(M)\right)$ a linear operator. For an ONB $\left(f_{m}\right)_{m \in \mathbb{N}}$ of $Y$ and $m \in \mathbb{N}$, we set $B_{m}:=\mathrm{B}(\cdot) f_{m}$. Additionally, we assume that $B_{m}, m \in \mathbb{N}$, are bounded operators on $H^{1}(M)$ with

$$
\sum_{m=1}^{\infty}\left\|B_{m}\right\|_{\mathcal{L}\left(H^{1}\right)}^{2}<\infty
$$

and that $B_{m}$ is symmetric as operator in $L^{2}(M)$, i.e.

$$
\left(B_{m} u, v\right)_{L^{2}}=\left(u, B_{m} v\right)_{L^{2}}, \quad u, v \in H^{1}(M)
$$

In particular, we have $B \in \mathcal{L}\left(H^{1}(M), \operatorname{HS}\left(Y, H^{1}(M)\right)\right)$ and $\mu \in \mathcal{L}\left(H^{1}(M)\right)$ if we abbreviate 


$$
\mu(u):=-\frac{1}{2} \sum_{m=1}^{\infty} B_{m}^{2} u, \quad u \in H^{1}(M)
$$

We look at the following slight generalization of (1.1) in the Itô form

$$
\left\{\begin{aligned}
\mathrm{d} u(t) & =\left(\mathrm{i} \Delta_{g} u(t)-\mathrm{i} \lambda|u(t)|^{\alpha-1} u(t)+\mu(u(t))\right) \mathrm{d} t-\mathrm{i} B u(t) \mathrm{d} W(t), \quad t \in(0, T), \\
u(0) & =u_{0} .
\end{aligned}\right.
$$

In the introduction, we used that the process

$$
W=\sum_{m=1}^{\infty} f_{m} \beta_{m}
$$

with a sequence $\left(\beta_{m}\right)_{m \in \mathbb{N}}$ of independent Brownian motions is a cylindrical Wiener process in $Y$, see [25], Proposition 4.7, and the identity

$$
-\mathrm{i} B u(t) \circ \mathrm{d} W(t)=-\mathrm{i} B u(t) \mathrm{d} W(t)+\mu(u(t)) \mathrm{d} t,
$$

which relates Itô and Stratonovich noise. For the sake of simplicity, we restricted ourselves to the special case of multiplication operators

$$
B_{m} u=e_{m} u, u \in H^{1}(M) .
$$

with real valued functions $e_{m}$ satisfying

$$
\sum_{m=1}^{\infty}\left(\left\|\nabla e_{m}\right\|_{L^{3}}+\left\|e_{m}\right\|_{L^{\infty}}\right)^{2}<\infty
$$

We want to justify that they fit in Assumption 2.1. The Sobolev embedding $H^{1}(M) \hookrightarrow$ $L^{6}(M)$ and the Hölder inequality yield

$$
\begin{aligned}
\left\|\nabla\left(e_{m} u\right)\right\|_{L^{2}} & \leq\left\|u \nabla e_{m}\right\|_{L^{2}}+\left\|e_{m} \nabla u\right\|_{L^{2}} \leq\left\|\nabla e_{m}\right\|_{L^{3}}\|u\|_{L^{6}}+\left\|e_{m}\right\|_{L^{\infty}}\|\nabla u\|_{L^{2}} \\
& \lesssim\left(\left\|\nabla e_{m}\right\|_{L^{3}}+\left\|e_{m}\right\|_{L^{\infty}}\right)\|u\|_{H^{1}}, u \in H^{1}(M) .
\end{aligned}
$$

Thus,

$$
\begin{aligned}
& \left\|B_{m} u\right\|_{H^{1}} \approx\left\|e_{m} u\right\|_{L^{2}}+\left\|\nabla\left(e_{m} u\right)\right\|_{L^{2}} \\
& \lesssim\left(\left\|\nabla e_{m}\right\|_{L^{3}}+\left\|e_{m}\right\|_{L^{\infty}}\right)\|u\|_{H^{1}}, u \in H^{1}(M) .
\end{aligned}
$$


Note that the existence-Theorem from [9] additionally needs the assumptions $B_{m} \in$ $\mathcal{L}\left(L^{2}(M)\right) \cap \mathcal{L}\left(L^{\alpha+1}(M)\right)$ with

$$
\sum_{m=1}^{\infty}\left\|B_{m}\right\|_{\mathcal{L}\left(L^{2}\right)}^{2}<\infty, \quad \sum_{m=1}^{\infty}\left\|B_{m}\right\|_{\mathcal{L}\left(L^{\alpha+1}\right)}^{2}<\infty
$$

But in our example of multiplication operators, this assumption is implied by (2.5). In the first Definition, we explain two solution concepts for problem (1.1).

Definition 2.2 Let $T>0$ and $u_{0} \in H^{1}(M)$.

(a) A martingale solution to Problem (1.1) is a system $(\Omega, \mathcal{F}, \mathbb{P}, W, \mathbb{F}, u)$ with

- A probability space $(\Omega, \mathcal{F}, \mathbb{P})$

- A $Y$-valued cylindrical Wiener $W$ process on $\Omega$;

- A filtration $\mathbb{F}=\left(\mathcal{F}_{t}\right)_{t \in[0, T]}$ with the usual conditions;

- A continuous, $\mathbb{F}$-adapted process with values in $H^{-1}(M)$ such that almost all paths are in $C_{w}\left([0, T], H^{1}(M)\right)$ and $u \in L^{2}\left(\Omega \times[0, T], H^{1}(M)\right)$;

such that the equation

$$
\begin{aligned}
& u(t)=u_{0}+\int_{0}^{t}\left[\mathrm{i} \Delta_{g} u(s)-\mathrm{i} \lambda|u(s)|^{\alpha-1} u(s)+\mu(u(s))\right] \mathrm{d} s \\
& -\mathrm{i} \int_{0}^{t} B u(s) \mathrm{d} W(s)
\end{aligned}
$$

holds $\mathbb{P}$-almost surely in $H^{-1}(M)$ for all $t \in[0, T]$.

(b) Given a probability space $(\Omega, \mathcal{F}, \mathbb{P})$, a $Y$-valued cylindrical Wiener $W$ process on $\Omega$, and a filtration $\mathbb{F}=\left(\mathcal{F}_{t}\right)_{t \in[0, T]}$ with the usual conditions, a strong solution to Problem (1.1) is a continuous, $\mathbb{F}$-adapted process with values in $H^{-1}(M)$ such that almost all paths are in $C_{w}\left([0, T], H^{1}(M)\right), u \in L^{2}\left(\Omega \times[0, T], H^{1}(M)\right)$ and (2.7) holds almost surely in $H^{-1}(M)$ for all $t \in[0, T]$.

Remark 2.3 For $\alpha \in(1,3]$, the solution is almost surely continuous in $L^{2}(M)$. Indeed, this follows from the following mild form of the Itô Eq. (2.7),

$$
\begin{aligned}
& u(t)=e^{\mathrm{i} t \Delta_{g}} u_{0}+\int_{0}^{t} e^{\mathrm{i}(t-s) \Delta_{g}}\left[-\mathrm{i} \lambda|u(s)|^{\alpha-1} u(s)+\mu(u(s))\right] \mathrm{d} s \\
& -\mathrm{i} \int_{0}^{t} e^{\mathrm{i}(t-s) \Delta_{g}} B u(s) \mathrm{d} W(s)
\end{aligned}
$$

almost surely for all $t \in[0, T]$, see for example the proof of Proposition 3.1 in a similar situation, since the nonlinearity with $\alpha \in(1,3]$ maps $H^{1}(M)$ to $L^{2}(M)$ by the Sobolev embedding $H^{1}(M) \hookrightarrow L^{2 \alpha}(M)$.

In the following definition, we fix different notions of uniqueness. As we have seen in the previous remark, it makes sense to define uniqueness by comparing solutions in $C\left([0, T], L^{2}(M)\right)$. 
Definition 2.4 (a) We say that the solutions to Problem (1.1) are pathwise unique in $L^{2}\left(\Omega ; L^{\infty}\left(0, T ; H^{1}(M)\right)\right)$, if and only if given two martingale solutions $\left(\Omega, \mathcal{F}, \mathbb{P}, W, \mathbb{F}, u_{j}\right)$ with $u_{j} \in L^{2}\left(\Omega ; L^{\infty}\left(0, T ; H^{1}(M)\right)\right)$ for $j=1,2$, to Problem (1.1), we have $u_{1}(t)=u_{2}(t)$ almost surely in $L^{2}(M)$ for all $t \in[0, T]$.

(b) We say that the solutions to Problem (1.1) are unique in law in $L^{2}\left(\Omega ; L^{\infty}(0, T\right.$; $\left.H^{1}(M)\right)$ ), if given two martingale solutions $\left(\Omega_{j}, \mathcal{F}_{j}, \mathbb{P}_{j}, W_{j}, \mathbb{F}_{j}, u_{j}\right)$ with $u_{j}(0)=u_{0}$ and $u_{j} \in L^{2}\left(\Omega ; L^{\infty}\left(0, T ; H^{1}(M)\right)\right)$ for $j=1,2$, to Problem (1.1), we have $\mathbb{P}_{1}^{u_{1}}=\mathbb{P}_{2}^{u_{2}}$ on $C\left([0, T], L^{2}(M)\right)$.

We continue with some auxiliary results which are either well-known or due to Burq, Gérard and Tzvetkov, [7]. The first Lemma gives us an estimate for the nonlinear term in Problem (1.1).

Lemma 2.5 Let $q \in[2,6]$ and $r \in(1, \infty)$ with $\frac{1}{r^{\prime}}=\frac{1}{2}+\frac{\alpha-1}{q}$. Then, we have

$$
\left\||u|^{\alpha-1} u\right\|_{H^{1, r^{\prime}}} \lesssim\|u\|_{H^{1}}^{\alpha}, u \in H^{1}(M) .
$$

Proof See [11], Lemma III.1.4.

The following Lemma deals with a Littlewood-Paley type decomposition of $L^{p}(M)$ for $p \in[2, \infty)$.

Lemma 2.6 Let $\psi \in C_{c}^{\infty}(\mathbb{R}), \varphi \in C_{c}^{\infty}(\mathbb{R} \backslash\{0\})$ with

$$
1=\psi(\lambda)+\sum_{k=1}^{\infty} \varphi\left(2^{-k} \lambda\right), \lambda \in \mathbb{R}
$$

Then, we have

$$
\|f\|_{L^{2}} \approx\left(\left\|\psi\left(\Delta_{g}\right) f\right\|_{L^{2}}^{2}+\sum_{k=1}^{\infty}\left\|\varphi\left(2^{-k} \Delta_{g}\right) f\right\|_{L^{2}}^{2}\right)^{\frac{1}{2}}, f \in L^{2}(M),
$$

and

$$
\|f\|_{L^{p}} \lesssim_{p}\left\|\psi\left(\Delta_{g}\right) f\right\|_{L^{p}}+\left(\sum_{k=1}^{\infty}\left\|\varphi\left(2^{-k} \Delta_{g}\right) f\right\|_{L^{p}}^{2}\right)^{\frac{1}{2}}, f \in L^{p}(M)
$$

for $p \in[2, \infty)$.

Proof Let $p \in(1, \infty)$. By [12], page 2, or [37] Theorem 4.1 and estimate (2.9) in a more general setting, we have

$$
\|f\|_{L^{p}} \approx\left\|\left(\left|\psi\left(\Delta_{g}\right) f\right|^{2}+\sum_{k=1}^{\infty}\left|\varphi\left(2^{-k} \Delta_{g}\right) f\right|^{2}\right)^{\frac{1}{2}}\right\|_{L^{p}}, \quad f \in L^{p}(M) .
$$

Hence, we get (2.9) by Fubini and (2.10) by Minkowski’s inequality. 
The previous Lemma indicates the importance of estimating operators of the form $\varphi\left(h^{2} \Delta_{g}\right)$ for $h \in(0,1]$. In the next Lemma, we state how they act in $L^{p}$-spaces and Sobolev spaces. Note that these kind of estimates are usually called Bernstein inequalities.

Lemma 2.7 (a) There is $C>0$ such that for all $1 \leq q \leq r \leq \infty$ and $\varphi \in C_{c}^{\infty}(\mathbb{R})$, the following inequality holds:

$$
\left\|\varphi\left(h^{2} \Delta_{g}\right) u\right\|_{L^{r}(M)} \leq C h^{d\left(\frac{1}{r}-\frac{1}{q}\right)}\left\|\varphi\left(h^{2} \Delta_{g}\right) u\right\|_{L^{q},}, \quad u \in L^{q}(M), \quad h \in(0,1] .
$$

(b) Let us assume that $p \in(1, \infty)$ and $s \geq 0$. Then, for every $\varphi \in C_{c}^{\infty}(\mathbb{R} \backslash\{0\})$, there is $C>0$ such that

$$
\left\|\varphi\left(h^{2} \Delta_{g}\right) u\right\|_{L^{p}} \leq C h^{s}\left\|\varphi\left(h^{2} \Delta_{g}\right) u\right\|_{H^{s, p},} \quad u \in H^{s, p}(M), \quad h \in(0,1] .
$$

Proof (a) See [7], Corollary 2.2. Let us emphasize that the fact that constant $C$ is independent of $q$ and $r$ follows from the proof given in [7].

(b) Throughout this proof, we w.l.o.g. assume $s>0$.

Moreover, we take $\tilde{\varphi} \in C_{c}^{\infty}(\mathbb{R} \backslash\{0\})$ with $\tilde{\varphi}=1$ on $\operatorname{supp}(\varphi)$ and define

$$
f_{h}:[0, \infty) \rightarrow \mathbb{R}, \quad f_{h}(t):=t^{-\frac{s}{2}} \tilde{\varphi}\left(-h^{2} t\right)
$$

for $h \in(0,1]$. Then, we have $\varphi\left(-h^{2} t\right)=f_{h}(t) t^{\frac{s}{2}} \varphi\left(-h^{2} t\right)$ for all $t \in[0, \infty)$ and $h \in(0,1]$. Furthermore, we obtain that $f_{h}$ satisfies the Mihlin condition

$$
\sup _{t \geq 0}\left|t^{k} f_{h}^{(k)}(t)\right| \lesssim h^{s}, \quad k \in \mathbb{N}_{0}, \quad h \in(0,1]
$$

Fact 2.20 in [47] and the Spectral Multiplier Theorem 7.6 in [24] hence imply

$$
\left\|f_{h}\left(-\Delta_{g}\right)\right\|_{\mathcal{L}\left(L^{1}, L^{1, \infty}\right)} \lesssim h^{s}, \quad h \in(0,1]
$$

Since we also have

$$
\left\|f_{h}\left(-\Delta_{g}\right)\right\|_{\mathcal{L}\left(L^{2}\right)} \leq \sup _{t \geq 0}\left|f_{h}(t)\right| \lesssim h^{s}, \quad h \in(0,1]
$$

by the Borel functional calculus for selfadjoint operators, the Marcinkiewitz Interpolation Theorem, see [27], Theorem 1.3.2, yields

$$
\left\|f_{h}\left(-\Delta_{g}\right)\right\|_{\mathcal{L}\left(L^{p}\right)} \lesssim h^{s}, \quad h \in(0,1]
$$

for $p \in(1,2]$. Since $f_{h}\left(-\Delta_{g}\right)$ is selfadjoint on $L^{2}(M)$, we obtain for $p \in(2, \infty)$ 


$$
\begin{aligned}
\left\|f_{h}\left(-\Delta_{g}\right)\right\|_{\mathcal{L}\left(L^{p}\right)} & =\sup _{u \in L^{p} \cap L^{2}:\|u\|_{L^{p}} \leq 1} \sup _{v \in L^{p^{\prime}} \cap L^{2}:\|v\|_{L^{p^{\prime}}} \leq 1}\left|\left(f_{h}\left(-\Delta_{g}\right) u, v\right)_{L^{2}}\right| \\
& =\sup _{v \in L^{p^{\prime}} \cap L^{2}:\|v\|_{L^{p^{\prime}}} \leq 1} \sup _{u \in L^{p} \cap L^{2}:\|u\|_{L^{p}} \leq 1}\left|\left(u, f_{h}\left(-\Delta_{g}\right) v\right)_{L^{2}}\right| \\
& =\left\|f_{h}\left(-\Delta_{g}\right)\right\|_{\mathcal{L}\left(L^{p^{\prime}}\right)} \lesssim h^{s}, \quad h \in(0,1] .
\end{aligned}
$$

For every $p \in(1, \infty)$, we therefore get

$$
\begin{aligned}
\left\|\varphi\left(h^{2} \Delta_{g}\right) u\right\|_{L^{p}} & =\left\|f_{h}\left(-\Delta_{g}\right)\left(-\Delta_{g}\right)^{\frac{s}{2}} \varphi\left(h^{2} \Delta_{g}\right) u\right\|_{L^{p}} \lesssim h^{s}\left\|\left(-\Delta_{g}\right)^{\frac{s}{2}} \varphi\left(h^{2} \Delta_{g}\right) u\right\|_{L^{p}} \\
& \lesssim h^{s}\left\|\varphi\left(h^{2} \Delta_{g}\right) u\right\|_{H^{s, p}}, u \in H^{s, p}(M) .
\end{aligned}
$$

This completes the proof of Lemma 2.7.

Definition 2.8 A pair $(p, q) \in[2, \infty]^{2}$ is called Strichartz-admissible for the dimension $d \in \mathbb{N}$ if

$$
\frac{2}{q}+\frac{d}{p}=\frac{d}{2} \text { and }(q, p, d) \neq(2, \infty, 2) .
$$

In the remainder of the article, we will use the exponent pairs $(6,2)$ and $(2, \infty)$ which are Strichartz-admissible for $d=3$. We emphasize that the Strichartz estimates which involve the pair $(6,2)$ are often called the endpoint Strichartz estimates. We refer to the article [35] in which Keel and Tao treated the endpoint case for the first time. Next, we collect the spectrally localized Strichartz estimates from [7] which will be crucial in the proof of Proposition 3.1.

Lemma 2.9 Let $M$ be a compact Riemannian manifold of dimension $d \in \mathbb{N}$ and a pair $(p, q) \in[2, \infty]^{2}$ be Strichartz-admissible. Then, for any $\varphi \in C_{c}^{\infty}(\mathbb{R})$, there exist positive numbers $\beta>0$ and $C>0$ such that for $h \in(0,1]$ and any interval $J$ of length $|J| \leq \beta h$

$$
\left\|t \mapsto e^{\mathrm{i} t \Delta_{g}} \varphi\left(h^{2} \Delta_{g}\right) x\right\|_{L^{q}\left(J, L^{p}\right)} \leq C\|x\|_{L^{2}}, \quad x \in L^{2}(M) .
$$

Proof See [7], Proposition 2.9. The result follows from the dispersive estimate for the Schrödinger group from [7], Lemma 2.5, and an application of Keel-Tao's Theorem ( [35]) with $U(t)=e^{\mathrm{it} \Delta_{g}} \tilde{\varphi}\left(h^{2} \Delta_{g}\right) \mathbf{1}_{J}(t)$ for some $\tilde{\varphi} \in C_{c}^{\infty}(\mathbb{R})$ with $\tilde{\varphi}=1$ on $\operatorname{supp}(\varphi)$.

A similar result also holds for convolutions with the Schrödinger group.

Lemma 2.10 Let $M$ be a compact Riemannian manifold of dimension $d \in \mathbb{N}$ and $\left(p_{1}, q_{1}\right),\left(p_{2}, q_{2}\right) \in[2, \infty]^{2}$ be Strichartz-admissible. Then, for any $\varphi \in C_{c}^{\infty}(\mathbb{R})$, there is $\beta>0$ and $C>0$ such that for $h \in(0,1]$ and any interval $J$ of length $|J| \leq \frac{\beta h}{2}$

$$
\left\|t \mapsto \int_{-\infty}^{t} e^{\mathrm{i}(t-s) \Delta_{g}} \varphi\left(h^{2} \Delta_{g}\right) f(s) \mathrm{d} s\right\|_{L^{q_{1}\left(J, L^{\left.p_{1}\right)}\right.}} \leq C\left\|\varphi\left(h^{2} \Delta_{g}\right) f\right\|_{L^{q_{2}^{\prime}\left(J, L^{p_{2}^{\prime}}\right)}}
$$


Proof See [7], Lemma 3.4.

To prepare the next Lemma, we recall the following notation.

Notation 2.11 Let $E$ be a separable Banach space, $p \in[1, \infty), J \subset[0, \infty)$ an interval and $(\Omega, \mathcal{F}, \mathbb{P}, \mathbb{F})$ a filtered probability space. By $\mathcal{M}^{p}(J, X)$, we denote the space of $\mathbb{F}$-progressively measurable $E$-valued processes $\xi: J \times \Omega \rightarrow E$ with $\|\xi\|_{L^{p}(J \times \Omega, E)}<\infty$.

Adapting the proof of [10, Theorem 3.10] to the present situation, we obtain a new spectrally localized stochastic Strichartz estimate for the stochastic convolution processes with the Schrödinger group.

Lemma 2.12 Let $M$ be a compact Riemannian manifold of dimension 3. Let $\varphi, \tilde{\varphi} \in$ $C_{c}^{\infty}(\mathbb{R} \backslash\{0\})$ with $\tilde{\varphi}=1$ on $\operatorname{supp}(\varphi)$. Choose $\beta>0$ as in Lemma 2.9. Let $h \in(0,1]$ and $J \subset[0, T]$ be an interval of length $|J| \leq \beta h$ and $\chi_{h} \in C_{c}^{\infty}(\mathbb{R})$ with $\operatorname{supp}\left(\chi_{h}\right) \subset J$. For $B \in \mathcal{M}^{2}\left(J, \operatorname{HS}\left(Y, L^{2}\right)\right)$, we set

$$
G(t):=\int_{0}^{t} e^{\mathrm{i}(t-s) \Delta_{g}} \chi_{h}(s) \varphi\left(h^{2} \Delta_{g}\right) \mathrm{B}(s) \mathrm{d} W(s), \quad t \in J
$$

Then,

$$
\|G\|_{L^{2}\left(\Omega, L^{2}\left(J, L^{6}\right)\right)} \lesssim\left\|\tilde{\varphi}\left(h^{2} \Delta_{g}\right) B\right\|_{L^{2}\left(\Omega, L^{2}\left(J, \mathrm{HS}\left(Y, L^{2}\right)\right)\right)} .
$$

Proof We abbreviate

$$
F(t, s):=\mathbf{1}_{\{s \leq t\}} e^{\mathrm{i}(t-s) \Delta_{g}} \chi_{h}(s) \varphi\left(h^{2} \Delta_{g}\right) \mathrm{B}(s), \quad t, s \in J,
$$

and use the Burkholder-Davis-Gundy-inequality in the martingale type 2 Banach space $L^{2}\left(J, L^{6}\right)$, see for example [13], to estimate

$$
\|G\|_{L^{2}\left(\Omega, L^{2}\left(J, L^{6}\right)\right)}^{2}=\mathbb{E}\left\|\int_{J} F(\cdot, s) \mathrm{d} W(s)\right\|_{L^{2}\left(J, L^{6}\right)}^{2} \lesssim \mathbb{E} \int_{J}\|F(\cdot, s)\|_{\gamma\left(Y, L^{2}\left(J, L^{6}\right)\right)}^{2} \mathrm{~d} s
$$

Writing out the definition of $\gamma\left(Y, L^{2}\left(J, L^{6}\right)\right)$ and using $\varphi\left(h^{2} \Delta_{g}\right)=\varphi\left(h^{2} \Delta_{g}\right) \tilde{\varphi}\left(h^{2} \Delta_{g}\right)$, we get

$$
\begin{aligned}
\|F(\cdot, s)\|_{\gamma\left(Y, L^{2}\left(J, L^{6}\right)\right)}^{2} & =\tilde{\mathbb{E}}\left\|t \mapsto \sum_{m=1}^{\infty} \gamma_{m} \mathbf{1}_{\{s \leq t\}} e^{\mathrm{i}(t-s) \Delta_{g}} \chi_{h}(s) \varphi\left(h^{2} \Delta_{g}\right) \mathrm{B}(s) f_{m}\right\|_{L^{2}\left(J, L^{6}\right)}^{2} \\
& =\tilde{\mathbb{E}}\left\|t \mapsto \sum_{m=1}^{\infty} e^{\mathrm{i} t \Delta_{g}} \varphi\left(h^{2} \Delta_{g}\right)\left[\gamma_{m} e^{-\mathrm{i} s \Delta_{g}} \chi_{h}(s) \tilde{\varphi}\left(h^{2} \Delta_{g}\right) \mathrm{B}(s) f_{m}\right]\right\|_{L^{2}\left(J_{\geq s}, L^{6}\right)}^{2}
\end{aligned}
$$

where $\left(\gamma_{m}\right)_{m \in \mathbb{N}}$ is a sequence of i.i.d. $\mathcal{N}(0,1)$-Gaussians on some probability space $\tilde{\Omega}$. By Lemma 2.9, the operator $e^{\mathrm{i} \cdot \Delta_{g}} \varphi\left(h^{2} \Delta_{g}\right)$ is bounded from $L^{2}(M)$ to $L^{2}\left(J, L^{6}\right)$. 
Hence, we can take it out of the sum and obtain

$$
\begin{aligned}
\|F(\cdot, s)\|_{\gamma\left(Y, L^{2}\left(J, L^{6}\right)\right)}^{2} & \lesssim \tilde{\mathbb{E}}\left\|\sum_{m=1}^{\infty} \gamma_{m} e^{-\mathrm{i} s \Delta_{g}} \chi_{h}(s) \tilde{\varphi}\left(h^{2} \Delta_{g}\right) \mathrm{B}(s) f_{m}\right\|_{L^{2}}^{2} \\
& =\left\|e^{-\mathrm{i} s \Delta_{g}} \chi_{h}(s) \tilde{\varphi}\left(h^{2} \Delta_{g}\right) \mathrm{B}(s)\right\|_{\gamma\left(Y, L^{2}\right)}^{2} \\
& \approx\left\|e^{-\mathrm{i} s \Delta_{g}} \chi_{h}(s) \tilde{\varphi}\left(h^{2} \Delta_{g}\right) \mathrm{B}(s)\right\|_{\mathrm{HS}\left(Y, L^{2}\right)}^{2} \\
& \lesssim\left\|\chi_{h}(s) \tilde{\varphi}\left(h^{2} \Delta_{g}\right) \mathrm{B}(s)\right\|_{\mathrm{HS}\left(Y, L^{2}\right)}^{2} .
\end{aligned}
$$

Finally, inserting the last estimate in (2.12) yields

$$
\begin{aligned}
\|G\|_{L^{2}\left(\Omega, L^{2}\left(J, L^{6}\right)\right)}^{2} & \lesssim \mathbb{E} \int_{J}\left\|\chi_{h}(s) \tilde{\varphi}\left(h^{2} \Delta_{g}\right) \tilde{\varphi}\left(h^{2} \Delta_{g}\right) \mathrm{B}(s)\right\|_{\mathrm{HS}\left(Y, L^{2}\right)}^{2} \mathrm{~d} s \\
& \lesssim\left\|\tilde{\varphi}\left(h^{2} \Delta_{g}\right) B\right\|_{L^{2}\left(\Omega, L^{2}\left(J, \operatorname{HS}\left(Y, L^{2}\right)\right)\right)}^{2} .
\end{aligned}
$$

The proof of Lemma 2.12 is thus completed.

Lemma 2.13 Let $a \in(0, \infty), b \in(0,1)$, let $X_{k}: \Omega \rightarrow[0, \infty], k \in \mathbb{N}_{0}$, be random variables which satisfy $\mathbb{E} X_{k} \leq a b^{k}$ for all $k \in \mathbb{N}_{0}$. Then for every $\varepsilon \in(0,1)$ there exists a random variable $C: \Omega \rightarrow[0, \infty]$ with $\mathbb{P}(C<\infty)=1$ which satisfies $X_{k} \leq C b^{(1-\varepsilon) k}$ for all $k \in \mathbb{N}_{0}$.

Proof Let $C:=\sum_{j=0}^{\infty} \xi_{j}$, where $\xi_{j}, j \in \mathbb{N}_{0}$, are nonnegative random variables given by $\xi_{j}:=X_{j} b^{-(1-\varepsilon) j}$ for $j \in \mathbb{N}_{0}$. Then, $C$ is a non-negative random variable and from monotone convergence and the assumptions, we infer

$$
\begin{aligned}
\mathbb{E}[C] & =\sum_{j=0}^{\infty} \mathbb{E} \xi_{j}=\sum_{j=0}^{\infty} b^{-(1-\varepsilon) j} \mathbb{E} X_{j} \leq \sum_{j=0}^{\infty} b^{-(1-\varepsilon) j} a b^{j} \\
& =a \sum_{j=0}^{\infty} b^{\varepsilon j}=\frac{a}{1-b^{\varepsilon}}<\infty
\end{aligned}
$$

As a consequence, we obtain $\mathbb{P}(C<\infty)=1$ and for all $k \in \mathbb{N}_{0}$, we have

$$
X_{k}=\xi_{k} b^{(1-\varepsilon) k} \leq \sum_{j=0}^{\infty} \xi_{j} b^{(1-\varepsilon) k}=C b^{(1-\varepsilon) k}
$$

\section{Uniqueness}

In the following section, we will prove the pathwise uniqueness of solutions of (1.1). A key ingredient for this result is an $L_{t}^{2} L_{x}^{p}$-estimate for solutions for arbitrary large $p$ with moderate growth of the bound in $p$. 
Proposition 3.1 Let $d=3$ and $\alpha \in(1,3]$. Let $T>0$ and let $(\Omega, \mathcal{F}, \mathbb{P}, W, \mathbb{F}, u)$, where $\mathbb{F}=\left(\mathcal{F}_{t}\right)_{t \in[0, T]}$, be a martingale solution of Problem (1.1). Then, there are a measurable set $\Omega_{\infty} \subset \Omega$ with $\mathbb{P}\left(\Omega_{\infty}\right)=1$ and a random variable $\mathfrak{C}: \Omega \rightarrow[0, \infty]$ with $\mathfrak{C}<\infty$ on $\Omega_{\infty}$ such that for all $\omega \in \Omega_{\infty}, p \in[6, \infty)$ and intervals $J \subset[0, T]$, we have

$$
\|u(\cdot, \omega)\|_{L^{2}\left(J, L^{p}\right)} \leq \mathfrak{C}(\omega)\left(1+(|J| p)^{\frac{1}{2}}\right) .
$$

Let us emphasize that the random constant $\mathfrak{C}$ in the estimate is independent of $p$. We further remark that this estimate of $L^{p}$-norms is a substitute for the $L^{\infty}$-bound for solutions in the $2 D$-setting, see [9], and complements the inequality, for $p \in[1,6]$,

$$
\|u\|_{L^{2}\left(J, L^{p}\right)} \lesssim|J|^{\frac{1}{2}}\|u\|_{L^{\infty}\left(J, H^{1}\right)}<\infty \quad \text { a.s. }
$$

which we get from the Sobolev embedding and the energy estimate for martingale solutions. Before we start with the proof, we introduce an equidistant partition of the time interval.

Notation 3.2 Let $I=[a, b]$ with $0<a<b<\infty$. For $\rho>0$ and $N:=\left\lfloor\frac{b-a}{\rho}\right\rfloor$, i.e. $N=\max \left\{n \in \mathbb{N}: n \leq \frac{b-a}{\rho}\right\}$, the family $\left(I_{j}\right)_{j=0}^{N}$ defined by

$$
\begin{aligned}
I_{j}: & =[a+j \rho, a+(j+1) \rho], \quad j \in\{0, \ldots N-1\}, \\
I_{N}: & =[a+N \rho, b]
\end{aligned}
$$

is called the $\rho$-partition of $I$. Observe that

$$
\left|I_{j}\right| \leq \rho, \quad j=0, \ldots, N, \quad I=\bigcup_{j=0}^{N} I_{j}, \quad I_{j}^{\circ} \cap I_{k}^{\circ}=\emptyset, \quad j \neq k
$$

Proof of Proposition 3.1 Let us choose and fix $T>0$ and a martingale solution $(\Omega, \mathcal{F}, \mathbb{P}, W, \mathbb{F}, u)$ to Problem (1.1). We choose $\beta>0$ as in Lemma 2.9 and in Lemma 2.10 .

Step 1 Let us choose $h \in(0,1]$ and take the $\frac{\beta h}{4}$-partition $\left(I_{j}\right)_{j=0}^{N_{T, h}}$ of the interval $[0, T]$ in the sense of Notation 3.2. Furthermore, we define a cover $\left(I_{j}^{\prime}\right)_{j=0}^{N_{T, h}}$ of $\left(I_{j}\right)_{j=0}^{N_{T, h}}$, a sequence $\left(m_{j}\right)_{j=0}^{N_{T, h}}$ by

$$
I_{j}^{\prime}:=\left(I_{j}+\left[-\frac{\beta h}{8}, \frac{\beta h}{8}\right]\right) \cap[0, T], m_{j}:=\frac{j \beta h}{4}+\frac{\beta h}{8}, \quad j=0, \ldots, N_{T, h},
$$


and a sequence $\left(\chi_{I_{j}}\right)_{j=0}^{N_{T, h}} \subset C_{c}^{\infty}([0, \infty))$ by $\chi_{I_{j}}:=\chi\left((\beta h)^{-1}\left(\cdot-m_{j}\right)\right)$ for some fixed $\chi \in C_{c}^{\infty}(\mathbb{R})$ with $\mathbf{1}_{\left[-\frac{1}{8}, \frac{1}{8}\right]} \leq \chi \leq \mathbf{1}_{\left[-\frac{1}{4}, \frac{1}{4}\right]}$. Then, we have

$$
\chi_{I_{j}}=1 \quad \text { on } I_{j}, \quad \operatorname{supp}\left(\chi_{I_{j}}\right) \subset I_{j}^{\prime}, \quad\left\|\chi_{I_{j}}^{\prime}\right\|_{L^{\infty}(\mathbb{R})} \leq(\beta h)^{-1}\left\|\chi^{\prime}\right\|_{L^{\infty}(\mathbb{R})} .
$$

We fix $\varphi, \tilde{\varphi} \in C_{c}^{\infty}(\mathbb{R} \backslash\{0\})$ with $0 \leq \varphi, \tilde{\varphi} \leq 1$ and $\tilde{\varphi}=1$ on $\operatorname{supp}(\varphi)$. In order to localize the solution $u$ spectrally and in time, we set

$$
v_{I_{j}}(t)=\chi_{I_{j}}(t) \varphi\left(h^{2} \Delta_{g}\right) u(t), \quad t \in[0, T], \quad j=0, \ldots, N_{T, h},
$$

and apply the Itô formula to $\Phi_{j} \in C^{1,2}\left(I_{j}^{\prime} \times H^{-1}(M), H^{-3}(M)\right)$ defined by

$$
\Phi_{j}(s, x)=e^{\mathrm{i}(t-s) \Delta_{g}} \chi_{I_{j}}(s) \varphi\left(h^{2} \Delta_{g}\right) x, \quad s \in I_{j}^{\prime}, \quad x \in H^{-3}(M),
$$

to get the following mild form representation of $v_{I_{j}}$, for $j=1, \ldots, N_{T, h}$,

$$
\begin{aligned}
v_{I_{j}}(t)= & \Phi_{j}\left(t, \chi_{I_{j}}(t) \varphi\left(h^{2} \Delta_{g}\right) u(t)\right) \\
= & \int_{\min I_{j}^{\prime}}^{t}\left[-\mathrm{i} \Delta_{g} e^{\mathrm{i}(t-s) \Delta_{g}} \chi_{I_{j}}(s) \varphi\left(h^{2} \Delta_{g}\right) u(s)+e^{\mathrm{i}(t-s) \Delta_{g}} \chi_{I_{j}}^{\prime}(s) \varphi\left(h^{2} \Delta_{g}\right) u(s)\right] \mathrm{d} s \\
& +\int_{\min I_{j}^{\prime}}^{t} e^{\mathrm{i}(t-s) \Delta_{g}} \chi_{I_{j}}(s) \varphi\left(h^{2} \Delta_{g}\right)\left[\mathrm{i} \Delta_{g} u(s)-\mathrm{i} \lambda|u(s)|^{\alpha-1} u(s)+\mu(u(s))\right] \mathrm{d} s \\
& -\mathrm{i} \int_{\min I_{j}^{\prime}}^{t} e^{\mathrm{i}(t-s) \Delta_{g}} \chi_{I_{j}}(s) \varphi\left(h^{2} \Delta_{g}\right) B u(s) \mathrm{d} W(s) \\
= & \int_{\min I_{j}^{\prime}}^{t} e^{\mathrm{i}(t-s) \Delta_{g}} \chi_{I_{j}}^{\prime}(s) \varphi\left(h^{2} \Delta_{g}\right) u(s) \mathrm{d} s \\
& +\int_{\min I_{j}^{\prime}}^{t} e^{\mathrm{i}(t-s) \Delta_{g}} \chi_{I_{j}}(s) \varphi\left(h^{2} \Delta_{g}\right)\left[-\mathrm{i} \lambda|u(s)|^{\alpha-1} u(s)+\mu(u(s))\right] \mathrm{d} s \\
& -\mathrm{i} \int_{\min I_{j}^{\prime}}^{t} e^{\mathrm{i}(t-s) \Delta_{g}} \chi_{I_{j}}(s) \varphi\left(h^{2} \Delta_{g}\right) B u(s) \mathrm{d} W(s)
\end{aligned}
$$

in $H^{-3}(M)$ almost surely for all $t \in I_{j}^{\prime}$. Because of the regularity of each term (recall that $\alpha \leq 3$ ), the identity (3.3) holds in $L^{2}(M)$ almost surely for all $t \in I_{j}^{\prime}$. Analogously, we get

$$
\begin{aligned}
v_{I_{0}}(t)= & e^{\mathrm{i} t \Delta_{g}} v_{I_{0}}(0)+\int_{0}^{t} e^{\mathrm{i}(t-s) \Delta_{g}} \chi_{I_{0}}^{\prime}(s) \varphi\left(h^{2} \Delta_{g}\right) u(s) \mathrm{d} s \\
& +\int_{0}^{t} e^{\mathrm{i}(t-s) \Delta_{g}} \chi_{I_{0}}(s) \varphi\left(h^{2} \Delta_{g}\right)\left[-\mathrm{i} \lambda|u(s)|^{\alpha-1} u(s)+\mu(u(s))\right] \mathrm{d} s \\
& -\mathrm{i} \int_{0}^{t} e^{\mathrm{i}(t-s) \Delta_{g}} \chi_{I_{0}}(s) \varphi\left(h^{2} \Delta_{g}\right) B u(s) \mathrm{d} W(s)
\end{aligned}
$$


in $L^{2}(M)$ almost surely for all $t \in I_{0}^{\prime}$. We abbreviate

$$
G_{I_{j}, h}(t):=\int_{\min I_{j}^{\prime}}^{t} e^{\mathrm{i}(t-s) \Delta_{g}} \chi_{I_{j}}(s) \varphi\left(h^{2} \Delta_{g}\right) B u(s) \mathrm{d} W(s), \quad 0 \leq t \in[0, T] .
$$

We use the stochastic Strichartz estimate from Lemma 2.12, the properties of the partitions $\left(I_{j}\right)_{j=0}^{N_{T, h}}$ and $\left(I_{j}^{\prime}\right)_{j=0}^{N_{T, h}}$ and Lemma $\left.2.7 \mathrm{~b}\right)$ to estimate

$$
\begin{aligned}
\mathbb{E} \sum_{j=0}^{N_{T, h}}\left\|G_{I_{j}, h}\right\|_{L^{2}\left(I_{j}^{\prime}, L^{6}\right)}^{2} & \lesssim \mathbb{E} \sum_{j=0}^{N_{T, h}} \int_{I_{j}^{\prime}}\left\|\tilde{\varphi}\left(h^{2} \Delta_{g}\right) \mathrm{B}(u(s))\right\|_{\mathrm{HS}\left(Y, L^{2}\right)}^{2} \mathrm{~d} s \\
& \leq 2 \mathbb{E} \sum_{j=0}^{N_{T, h}} \int_{I_{j}}\left\|\tilde{\varphi}\left(h^{2} \Delta_{g}\right) \mathrm{B}(u(s))\right\|_{\mathrm{HS}\left(Y, L^{2}\right)}^{2} \mathrm{~d} s \\
& =2 \mathbb{E} \int_{0}^{T}\left\|\tilde{\varphi}\left(h^{2} \Delta_{g}\right) \mathrm{B}(u(s))\right\|_{\mathrm{HS}\left(Y, L^{2}\right)}^{2} \mathrm{~d} s \\
& \lesssim h^{2} \mathbb{E} \int_{0}^{T}\left\|\tilde{\varphi}\left(h^{2} \Delta_{g}\right) \mathrm{B}(u(s))\right\|_{\mathrm{HS}\left(Y, H^{1}\right)}^{2} \mathrm{~d} s
\end{aligned}
$$

Since $\tilde{\varphi}\left(h^{2} \Delta_{g}\right)$ is a contractive operator from $H^{1}(M)$ to $H^{1}(M)\left(\right.$ recall $\left.\|\tilde{\varphi}\|_{\infty} \leq 1\right)$ and $B$ is bounded from $H^{1}(M)$ to $\operatorname{HS}\left(Y, H^{1}(M)\right)$ by Assumption 2.1, we conclude

$$
\mathbb{E} \sum_{j=0}^{N_{T, h}}\left\|G_{I_{j}, h}\right\|_{L^{2}\left(I_{j}^{\prime}, L^{6}\right)}^{2} \lesssim h^{2} \mathbb{E} \int_{0}^{T}\|u(s)\|_{H^{1}}^{2} \mathrm{~d} s, \quad h \in(0,1)
$$

We emphasize that in view of Lemma 2.12 the implicit constant in (3.6) is independent of $h \in(0,1)$. Consequently, Lemma 2.13 shows that for any $\varepsilon \in(0,1)$ there is a random variable $C: \Omega \rightarrow[0, \infty]$ with $C<\infty$ almost surely such that

$$
\sum_{j=0}^{N_{T, 2^{-k / 2}}}\left\|G_{I_{j}, 2^{-k / 2}}\right\|_{L^{2}\left(I_{j}^{\prime}, L^{6}\right)}^{2} \leq 2^{-k(1-\varepsilon)} C \text { on } \Omega \quad \text { for all } k \in \mathbb{N}_{0}
$$

Step 2 Now, let us choose and fix an interval $J \subset[0, T]$, let $\varepsilon \in(0,1 / 2), k \in$ $\mathbb{N}_{0}$ and $h=2^{-k / 2}$. Let us define $\Omega_{k}$ as the intersection of the full probability sets from (3.3), (3.4) and (3.7) for $h=2^{-k / 2}$ and the assumption that $u_{j} \in$ $L^{\infty}\left(0, T ; H^{1}(M)\right)$ almost surely. Moreover, we set $\Omega_{\infty}:=\bigcap_{j=0}^{\infty} \Omega_{j}$ and notice that $\mathbb{P}\left(\Omega_{\infty}\right)=1$. Let us next choose and fix a path $\omega \in \Omega_{\infty}$. In the rest of the argument, we skip the dependence on $\omega$ to keep the notation simple. Let us pick those intervals $J_{0}, \ldots, J_{N}$ from the partition $\left(I_{j}\right)_{j=0}^{N_{T, h}}$ which cover the given 
interval $J$. The associated intervals in $\left(I_{j}^{\prime}\right)_{j=0}^{N}$ will be denoted by $J_{0}^{\prime}, \ldots, J_{N}^{\prime}$. From (3.3) and (3.5) we deduce for $j=1, \ldots, N$ that

$$
\begin{aligned}
v_{J_{j}}(t)= & \int_{\min J_{j}^{\prime}}^{t} e^{\mathrm{i}(t-s) \Delta_{g}} \varphi\left(h^{2} \Delta_{g}\right)\left[\chi_{J_{j}}^{\prime}(s) u(s)+\chi_{J_{j}}(s) \mu(u(s))\right] \mathrm{d} s \\
& -\mathrm{i} \lambda \int_{\min J_{j}^{\prime}}^{t} e^{\mathrm{i}(t-s) \Delta_{g}} \chi_{J_{j}}(s) \varphi\left(h^{2} \Delta_{g}\right)|u(s)|^{\alpha-1} u(s) \mathrm{d} s-\mathrm{i} G_{J_{j}, h}(t)
\end{aligned}
$$

holds in $L^{2}(M)$ almost surely for all $t \in I_{j}^{\prime}$. Note that (3.1) implies $\left|J_{j}^{\prime}\right| \leq \frac{\beta h}{2}$. Due to the choice of $\beta>0$, we can apply Lemma 2.10 with the Strichartz-admissible pairs $(6,2)$ and $(2, \infty)$. Hence, we obtain for $j=1, \ldots, N$

$$
\begin{aligned}
\| t & \mapsto \int_{\min J_{j}^{\prime}}^{t} e^{\mathrm{i}(t-s) \Delta_{g}} \varphi\left(h^{2} \Delta_{g}\right)\left[\chi_{J_{j}}^{\prime}(s) u(s)+\chi_{J_{j}}(s) \mu(u(s))\right] \mathrm{d} s \|_{L^{2}\left(J_{j}^{\prime}, L^{6}\right)} \\
& \lesssim\left\|\varphi\left(h^{2} \Delta_{g}\right)\left[\chi_{J_{j}}^{\prime} u+\chi_{J_{j}} \mu(u)\right]\right\|_{L^{1}\left(J_{j}^{\prime}, L^{2}\right)}
\end{aligned}
$$

and

$$
\begin{aligned}
\| t & \mapsto \int_{\min J_{j}^{\prime}}^{t} e^{\mathrm{i}(t-s) \Delta_{g}} \varphi\left(h^{2} \Delta_{g}\right) \chi_{J_{j}}(s)|u(s)|^{\alpha-1} u(s) \mathrm{d} s \|_{L^{2}\left(J_{j}^{\prime}, L^{6}\right)} \\
& \lesssim\left\|\varphi\left(h^{2} \Delta_{g}\right) \chi_{J_{j}}|u|^{\alpha-1} u\right\|_{L^{2}\left(J_{j}^{\prime}, L^{\frac{6}{5}}\right)} \cdot
\end{aligned}
$$

Let us emphasize that the implicit constants in these estimates are independent of $h$ and $\omega$. Combining the latter estimates with (3.8) yields

$$
\begin{aligned}
& \left\|v_{J_{j}}\right\|_{L^{2}\left(J_{j}, L^{6}\right)} \leq\left\|v_{J_{j}}\right\|_{L^{2}\left(J_{j}^{\prime}, L^{6}\right)} \lesssim\left\|\chi_{J_{j}}^{\prime} \varphi\left(h^{2} \Delta_{g}\right) u\right\|_{L^{1}\left(J_{j}^{\prime}, L^{2}\right)} \\
& \quad+\left\|\chi_{J_{j}} \varphi\left(h^{2} \Delta_{g}\right)|u|^{\alpha-1} u\right\|_{L^{2}\left(J_{j}^{\prime}, L^{\frac{6}{5}}\right)} \\
& \quad+\left\|\chi_{J_{j}} \varphi\left(h^{2} \Delta_{g}\right) \mu(u)\right\|_{L^{1}\left(J_{j}^{\prime}, L^{2}\right)}+\left\|G_{J_{j}, h}\right\|_{L^{2}\left(J_{j}^{\prime}, L^{6}\right)}
\end{aligned}
$$

for $j=1, \ldots, N$. Using similar arguments and taking into account Lemma 2.9 for the evolution of the initial value, we deduce

$$
\begin{aligned}
\left\|v_{J_{0}}\right\|_{L^{2}\left(J_{0}, L^{6}\right)} \leq\left\|v_{J_{0}}\right\|_{L^{2}\left(J_{0}^{\prime}, L^{6}\right)} \lesssim & \left\|v_{J_{0}}\left(\min J_{0}^{\prime}\right)\right\|_{L^{2}}+\left\|\chi_{J_{0}}^{\prime} \varphi\left(h^{2} \Delta_{g}\right) u\right\|_{L^{1}\left(J_{0}^{\prime}, L^{2}\right)} \\
& +\left\|\chi_{J_{0}} \varphi\left(h^{2} \Delta_{g}\right)|u|^{\alpha-1} u\right\|_{L^{2}\left(J_{0}^{\prime}, L^{\frac{6}{5}}\right)} \\
& +\left\|\chi_{J_{0}} \varphi\left(h^{2} \Delta_{g}\right) \mu(u)\right\|_{L^{1}\left(J_{0}^{\prime}, L^{2}\right)} \\
& +\left\|G_{J_{0}, h}\right\|_{L^{2}\left(J_{0}^{\prime}, L^{6}\right)}
\end{aligned}
$$


Note that $v_{J_{0}}\left(\min J_{0}^{\prime}\right)=0$ if $I_{0} \neq J_{0}$. Next, we estimate the terms on the right hand side of (3.9) and (3.10). By properties (3.2), Lemma 2.7 b) and the Hölder inequality with $\left|J_{j}^{\prime}\right| \lesssim h$, we get

$$
\begin{aligned}
\left\|\chi_{J_{j}}^{\prime} \varphi\left(h^{2} \Delta_{g}\right) u\right\|_{L^{1}\left(J_{j}^{\prime}, L^{2}\right)} & \lesssim h^{-1}\left\|\varphi\left(h^{2} \Delta_{g}\right) u\right\|_{L^{1}\left(J_{j}^{\prime}, L^{2}\right)} \lesssim\left\|\varphi\left(h^{2} \Delta_{g}\right) u\right\|_{L^{1}\left(J_{j}^{\prime}, H^{1}\right)} \\
& \lesssim h^{\frac{1}{2}}\left\|\varphi\left(h^{2} \Delta_{g}\right) u\right\|_{L^{2}\left(J_{j}^{\prime}, H^{1}\right)}
\end{aligned}
$$

The Hölder inequality with $\left|J_{j}^{\prime}\right| \lesssim h$, Lemma $2.7 \mathrm{~b}$ ) and the boundedness of the operators $\varphi\left(h^{2} \Delta_{g}\right)$ and $\mu$ in $H^{1}(M)$ yield

$$
\begin{aligned}
\left\|\chi_{J_{j}} \varphi\left(h^{2} \Delta_{g}\right) \mu(u)\right\|_{L^{1}\left(J_{j}^{\prime}, L^{2}\right)} & \lesssim h\left\|\chi_{J_{j}} \varphi\left(h^{2} \Delta_{g}\right) \mu(u)\right\|_{L^{\infty}\left(J_{j}^{\prime}, L^{2}\right)} \\
& \leq h\left\|\varphi\left(h^{2} \Delta_{g}\right) \mu(u)\right\|_{L^{\infty}\left(0, T ; L^{2}\right)} \\
& \lesssim h^{2}\left\|\varphi\left(h^{2} \Delta_{g}\right) \mu(u)\right\|_{L^{\infty}\left(0, T ; H^{1}\right)} \\
& \lesssim h^{2}\|u\|_{L^{\infty}\left(0, T ; H^{1}\right)} .
\end{aligned}
$$

Next we apply Lemma 2.5 with $r^{\prime}=\frac{6}{\alpha+2}$ and $q=6$. Since $\alpha \leq 3$ we have $r^{\prime} \geq \frac{6}{5}$ and hence we obtain the following estimate

$$
\left\||v|^{\alpha-1} v\right\|_{H^{1, \frac{6}{5}}} \lesssim\left\||v|^{\alpha-1} v\right\|_{H^{1, \frac{6}{\alpha+2}}} \lesssim\|v\|_{H^{1}}^{\alpha}, \quad v \in H^{1}(M) .
$$

Moreover, note that $\sup _{h \in(0,1)}\left\|\varphi\left(h^{2} \Delta_{g}\right)\right\|_{\mathcal{L}\left(H^{1, \frac{6}{5}}\right)}<\infty$. Together with (3.11), the Hölder inequality, and Lemma 2.7 b), this implies

$$
\begin{aligned}
\left\|\chi_{J_{j}} \varphi\left(h^{2} \Delta_{g}\right)|u|^{\alpha-1} u\right\|_{L^{2}\left(J_{j}^{\prime}, L^{\frac{6}{5}}\right)} & \lesssim h^{\frac{1}{2}}\left\|\varphi\left(h^{2} \Delta_{g}\right)|u|^{\alpha-1} u\right\|_{L^{\infty}\left(0, T ; L^{\frac{6}{5}}\right)} \\
& \lesssim h^{\frac{3}{2}}\left\|\varphi\left(h^{2} \Delta_{g}\right)|u|^{\alpha-1} u\right\|_{L^{\infty}\left(0, T ; H^{1, \frac{6}{5}}\right)} \\
& \lesssim h^{\frac{3}{2}}\left\||u|^{\alpha-1} u\right\|_{L^{\infty}\left(0, T ; H^{1, \frac{6}{5}}\right)} \lesssim h^{\frac{3}{2}}\|u\|_{L^{\infty}\left(0, T ; H^{1}\right)}^{\alpha},
\end{aligned}
$$

where we again emphasize that the implicit constants in the inequalities above and below are independent of $h \in(0,1)$. Inserting the last three estimates in (3.9) and (3.10) yields for $j=1, \ldots, N$

$$
\begin{gathered}
\left\|v_{J_{j}}\right\|_{L^{2}\left(J_{j}, L^{6}\right)} \lesssim h^{\frac{1}{2}}\left\|\varphi\left(h^{2} \Delta_{g}\right) u\right\|_{L^{2}\left(J_{j}^{\prime}, H^{1}\right)}+h^{\frac{3}{2}}\|u\|_{L^{\infty}\left(0, T ; H^{1}\right)}^{\alpha} \\
+h^{2}\|u\|_{L^{\infty}\left(0, T ; H^{1}\right)}+\left\|G_{J_{j}, h}\right\|_{L^{2}\left(J_{j}^{\prime}, L^{6}\right)}, \\
\left\|v_{J_{0}}\right\|_{L^{2}\left(J_{0}, L^{6}\right) \lesssim} h\left\|\varphi\left(h^{2} \Delta_{g}\right) u\left(\min J_{0}^{\prime}\right)\right\|_{H^{1}}+h^{\frac{1}{2}}\left\|\varphi\left(h^{2} \Delta_{g}\right) u\right\|_{L^{2}\left(J_{0}^{\prime}, H^{1}\right)} \\
+h^{\frac{3}{2}}\|u\|_{L^{\infty}\left(0, T ; H^{1}\right)}^{\alpha} \\
+h^{2}\|u\|_{L^{\infty}\left(0, T ; H^{1}\right)}+\left\|G_{J_{0}, h}\right\|_{L^{2}\left(J_{0}^{\prime}, L^{6}\right)} .
\end{gathered}
$$


We square the estimates (3.12) and (3.13) and sum them up over $j \in\{0, \ldots, N\}$. Since $\chi_{J_{j}}=1$ on $J_{j}$, by using (3.7) and $N \leq N_{T, h}=\left\lfloor\frac{4 T}{\beta h}\right\rfloor$, we conclude that

$$
\begin{aligned}
& \left\|\varphi\left(h^{2} \Delta_{g}\right) u\right\|_{L^{2}\left(J, L^{6}\right)}^{2} \leq \sum_{j=0}^{N}\left\|\chi_{J_{j}} \varphi\left(h^{2} \Delta_{g}\right) u\right\|_{L^{2}\left(J_{j}, L^{6}\right)}^{2}=\sum_{j=0}^{N}\left\|v_{J_{j}}\right\|_{L^{2}\left(J_{j}, L^{6}\right)}^{2} \\
& \lesssim h^{2}\left\|\varphi\left(h^{2} \Delta_{g}\right) u\left(\min J_{0}^{\prime}\right)\right\|_{H^{1}}^{2} \\
& +\sum_{j=0}^{N}\left[h\left\|\varphi\left(h^{2} \Delta_{g}\right) u\right\|_{L^{2}\left(J_{j}^{\prime}, H^{1}\right)}^{2}+h^{3}\|u\|_{L^{\infty}\left(0, T ; H^{1}\right)}^{2 \alpha}\right] \\
& +\sum_{j=0}^{N}\left[h^{4}\|u\|_{L^{\infty}\left(0, T ; H^{1}\right)}^{2}\right]+\sum_{j=0}^{N_{T, h}}\left\|G_{I_{j}, h}\right\|_{L^{2}\left(I_{j}^{\prime}, L^{6}\right)}^{2} \\
& \lesssim h^{2}\left\|\varphi\left(h^{2} \Delta_{g}\right) u\left(\min J_{0}^{\prime}\right)\right\|_{H^{1}}^{2} \\
& +\sum_{j=0}^{N}\left[h\left\|\varphi\left(h^{2} \Delta_{g}\right) u\right\|_{L^{2}\left(J_{j}^{\prime}, H^{1}\right)}^{2}+h^{3}\|u\|_{L^{\infty}\left(0, T ; H^{1}\right)}^{2 \alpha}\right] \\
& +\sum_{j=0}^{N}\left[h^{4}\|u\|_{L^{\infty}\left(0, T ; H^{1}\right)}^{2}\right]+h^{2(1-\varepsilon)} C \\
& \lesssim h^{2}\left\|\varphi\left(h^{2} \Delta_{g}\right) u\left(\min J_{0}^{\prime}\right)\right\|_{H^{1}}^{2}+h \sum_{j=0}^{N}\left\|\varphi\left(h^{2} \Delta_{g}\right) u\right\|_{L^{2}\left(J_{j}^{\prime}, H^{1}\right)}^{2} \\
& +h^{2}\|u\|_{L^{\infty}\left(0, T ; H^{1}\right)}^{2 \alpha}+h^{3}\|u\|_{L^{\infty}\left(0, T ; H^{1}\right)}^{2}+h^{2(1-\varepsilon)} C .
\end{aligned}
$$

Below, we will use the following additional notation

$$
J_{N+1}:=\left(\bigcup_{j=0}^{N} J_{j}^{\prime}\right) \backslash\left(\bigcup_{j=0}^{N} J_{j}\right), \quad J^{h}:=\bigcup_{j=0}^{N+1} J_{j} .
$$

Since

$$
\begin{aligned}
& \sum_{j=0}^{N}\left\|\varphi\left(h^{2} \Delta_{g}\right) u\right\|_{L^{2}\left(J_{j}^{\prime}, H^{1}\right)}^{2} \leq 2 \sum_{j=0}^{N+1}\left\|\varphi\left(h^{2} \Delta_{g}\right) u\right\|_{L^{2}\left(J_{j}, H^{1}\right)}^{2} \\
& =2\left\|\varphi\left(h^{2} \Delta_{g}\right) u\right\|_{L^{2}\left(J^{h}, H^{1}\right)}^{2}
\end{aligned}
$$

we infer that

$$
\begin{aligned}
\left\|\varphi\left(h^{2} \Delta_{g}\right) u\right\|_{L^{2}\left(J, L^{6}\right)}^{2} \lesssim & h^{2}\left\|\varphi\left(h^{2} \Delta_{g}\right) u\left(\min J_{0}^{\prime}\right)\right\|_{H^{1}}^{2}+h\left\|\varphi\left(h^{2} \Delta_{g}\right) u\right\|_{L^{2}\left(J^{h}, H^{1}\right)}^{2} \\
& +h^{2}\|u\|_{L^{\infty}\left(0, T ; H^{1}\right)}^{2 \alpha}+h^{3}\|u\|_{L^{\infty}\left(0, T ; H^{1}\right)}^{2}+h^{2(1-\varepsilon)} C .
\end{aligned}
$$


Let now choose and fix $p \geq 6$. Since $u \in L^{\infty}\left(0, T ; H^{1}(M)\right)$, by Lemma 2.7 a) we deduce for $h=2^{-k / 2}, k \in \mathbb{N}_{0}$, that

$$
\begin{aligned}
\left\|\varphi\left(h^{2} \Delta_{g}\right) u\right\|_{L^{2}\left(J, L^{p}\right) \lesssim} \lesssim & h^{3\left(\frac{1}{p}-\frac{1}{6}\right)}\left\|\varphi\left(h^{2} \Delta_{g}\right) u\right\|_{L^{2}\left(J, L^{6}\right)} \\
\lesssim & h^{\frac{3}{p}+\frac{1}{2}}\left\|\varphi\left(h^{2} \Delta_{g}\right) u\left(\min J_{0}^{\prime}\right)\right\|_{H^{1}}+h^{\frac{3}{p}}\left\|\varphi\left(h^{2} \Delta_{g}\right) u\right\|_{L^{2}\left(J^{h}, H^{1}\right)} \\
& +h^{\frac{3}{p}+\frac{1}{2}}\|u\|_{L^{\infty}\left(0, T ; H^{1}\right)}^{\alpha}+h^{\frac{3}{p}+1}\|u\|_{L^{\infty}\left(0, T ; H^{1}\right)}+h^{\frac{3}{p}+\frac{1}{2}-\varepsilon} C \\
\lesssim & h^{\frac{3}{p}+\frac{1}{2}}+h^{\frac{3}{p}}\left\|\varphi\left(h^{2} \Delta_{g}\right) u\right\|_{L^{2}\left(J^{h}, H^{1}\right)}+h^{\frac{3}{p}+\frac{1}{2}-\varepsilon}+h^{\frac{3}{p}+1}
\end{aligned}
$$

with the implicit constant being independent of $k$ and $p$ because the constant $C$ from Lemma 2.7 a) does not depend on $p$.

Step 3 In the last step, we use inequality (3.15) and the Littlewood-Paley theory to derive the estimate stated in the Proposition. To this end, we set $h_{k}:=2^{-\frac{k}{2}}$ and $k_{0}:=\min \left\{k:|J|>\frac{\beta h_{k}}{4}\right\}$. As in the previous step, we fix a path $\omega \in \Omega_{\infty}:=$ $\bigcap_{k=0}^{\infty} \Omega_{k}$ and, in the rest of the argument, we skip the dependence on $\omega$ to keep the notation simple. Moreover, we choose $\psi \in C_{c}^{\infty}(\mathbb{R}), \varphi \in C_{c}^{\infty}(\mathbb{R} \backslash\{0\})$ such that

$$
1=\psi(\lambda) u+\sum_{k=1}^{\infty} \varphi\left(2^{-k} \lambda\right), \quad \lambda \in \mathbb{R}
$$

Then, Lemma 2.6, the embedding $\ell^{1}(\mathbb{N}) \hookrightarrow \ell^{2}(\mathbb{N}),(3.15)$, and the hypothesis $\varepsilon \in$ $(0,1 / 2)$ from Step 2 imply that

$$
\begin{aligned}
\|u\|_{L^{2}\left(J, L^{p}\right)} \lesssim & \left\|\left(\left\|\psi\left(\Delta_{g}\right) u\right\|_{L^{p}}^{2}+\sum_{k=1}^{\infty}\left\|\varphi\left(2^{-k} \Delta_{g}\right) u\right\|_{L^{p}}^{2}\right)^{\frac{1}{2}}\right\|_{L^{2}(J)} \\
= & \left(\left\|\psi\left(\Delta_{g}\right) u\right\|_{L^{2}\left(J, L^{p}\right)}^{2}+\sum_{k=1}^{\infty}\left\|\varphi\left(2^{-k} \Delta_{g}\right) u\right\|_{L^{2}\left(J, L^{p}\right)}^{2}\right)^{\frac{1}{2}} \\
\leq & \left\|\psi\left(\Delta_{g}\right) u\right\|_{L^{2}\left(J, L^{p}\right)}+\sum_{k=1}^{\infty}\left\|\varphi\left(2^{-k} \Delta_{g}\right) u\right\|_{L^{2}\left(J, L^{p}\right)} \\
\lesssim & \left\|\psi\left(\Delta_{g}\right) u\right\|_{L^{2}\left(J, L^{p}\right)}+\sum_{k=1}^{k_{0}-1}\left\|\varphi\left(2^{-k} \Delta_{g}\right) u\right\|_{L^{2}\left(J, L^{p}\right)} \\
& +\sum_{k=k_{0}}^{\infty} 2^{-\frac{3 k}{2 p}}\left\|\varphi\left(2^{-k} \Delta_{g}\right) u\right\|_{L^{2}\left(J^{h}, H^{1}\right)} \\
& +\sum_{k=k_{0}}^{\infty}\left[2^{-\frac{k}{2}\left(\frac{3}{p}+\frac{1}{2}\right)}+2^{-\frac{k}{2}\left(\frac{3}{p}+1\right)}+2^{-\frac{k}{2}\left(\frac{3}{p}+\frac{1}{2}-\varepsilon\right)}\right] \\
\leq & \left\|\psi\left(\Delta_{g}\right) u\right\|_{L^{2}\left(J, L^{p}\right)}+\sum_{k=1}^{k_{0}-1}\left\|\varphi\left(2^{-k} \Delta_{g}\right) u\right\|_{L^{2}\left(J, L^{p}\right)}
\end{aligned}
$$




$$
\begin{aligned}
& +\sum_{k=k_{0}}^{\infty} 2^{-\frac{3 k}{2 p}}\left\|\varphi\left(2^{-k} \Delta_{g}\right) u\right\|_{L^{2}\left(J^{h_{k}, H^{1}}\right)}+\sum_{k=k_{0}}^{\infty}\left[2^{-\frac{k}{4}}+2^{-\frac{k}{2}}+2^{-\frac{k}{4}(1-2 \varepsilon)}\right] \\
\lesssim & \left\|\psi\left(\Delta_{g}\right) u\right\|_{L^{2}\left(J, L^{p}\right)}+\sum_{k=1}^{k_{0}-1}\left\|\varphi\left(2^{-k} \Delta_{g}\right) u\right\|_{L^{2}\left(J, L^{p}\right)} \\
& +\left(\sum_{k=k_{0}}^{\infty} 2^{-\frac{3 k}{p}}\right)^{\frac{1}{2}}\left(\sum_{k=k_{0}}^{\infty}\left\|\varphi\left(2^{-k} \Delta_{g}\right) u\right\|_{L^{2}\left(J^{h_{k}}, H^{1}\right)}^{2}\right)^{\frac{1}{2}}+1 .
\end{aligned}
$$

From Lemma 2.7 a) with $h=1$, we conclude

$$
\left\|\psi\left(\Delta_{g}\right) u\right\|_{L^{2}\left(J, L^{p}\right)} \lesssim\left\|\psi\left(\Delta_{g}\right) u\right\|_{L^{2}\left(J, L^{2}\right)} \lesssim\|u\|_{L^{2}\left(J, L^{2}\right)} \lesssim 1
$$

From Lemma 2.7 a) and the Sobolev embedding, we infer

$$
\begin{aligned}
\left\|\varphi\left(2^{-k} \Delta_{g}\right) u\right\|_{L^{2}\left(J, L^{p}\right)} & \lesssim 2^{-k\left(\frac{3}{2 p}-\frac{1}{4}\right)}\left\|\varphi\left(2^{-k} \Delta_{g}\right) u\right\|_{L^{2}\left(J, L^{6}\right)} \\
& \lesssim 2^{\frac{k}{4}}\left\|\varphi\left(2^{-k} \Delta_{g}\right) u\right\|_{L^{2}\left(J, H^{1}\right)}
\end{aligned}
$$

for $k \in\left\{1, \ldots, k_{0}-1\right\}$. From the definition of $k_{0}$, we have $|J| \approx 2^{-\frac{k_{0}}{2}}$. Thus, we get

$$
\begin{aligned}
\sum_{k=1}^{k_{0}-1}\left\|\varphi\left(2^{-k} \Delta_{g}\right) u\right\|_{L^{2}\left(J, L^{p}\right)} & \lesssim\left(\sum_{k=1}^{k_{0}-1} 2^{\frac{k}{2}}\right)^{\frac{1}{2}}\left(\sum_{k=1}^{k_{0}-1}\left\|\varphi\left(2^{-k} \Delta_{g}\right) u\right\|_{L^{2}\left(J, H^{1}\right)}^{2}\right)^{\frac{1}{2}} \\
& \lesssim 2^{\frac{k_{0}}{4}}\|u\|_{L^{2}\left(J, H^{1}\right)} \lesssim|J|^{-\frac{1}{2}}|J|^{\frac{1}{2}} \lesssim 1
\end{aligned}
$$

We proceed with the estimate of the sums over $k \geq k_{0}$. The fact that we have $J^{h_{k+1}} \subset$ $J^{h_{k}}$ for all $k \in \mathbb{N}$, leads to

$$
\begin{aligned}
\sum_{k=k_{0}}^{\infty}\left\|\varphi\left(2^{-k} \Delta_{g}\right) u\right\|_{L^{2}\left(J^{\left.h_{k}, H^{1}\right)}\right.}^{2} & =\sum_{k:|J|>\frac{\beta h_{k}}{4}}\left\|\varphi\left(2^{-k} \Delta_{g}\right) u\right\|_{L^{2}\left(J^{h_{k}, H^{1}}\right)}^{2} \\
& \leq \sum_{k:|J|>\frac{\beta h_{k}}{4}}\left\|\varphi\left(2^{-k} \Delta_{g}\right) u\right\|_{L^{2}\left(J^{\left.h_{0}, H^{1}\right)}\right.}^{2} \\
& \lesssim\|u\|_{L^{2}\left(J^{\left.h_{k_{0}}, H^{1}\right)}\right.}^{2} \leq \mid J^{h_{k_{0}} \mid\|u\|_{L^{\infty}\left(0, T ; H^{1}\right)}^{2}} .
\end{aligned}
$$

Using $\left|J^{h_{k_{0}}}\right| \leq 3 \frac{\beta h_{k_{0}}}{4}+|J| \leq 4|J|$ and $u \in L^{\infty}\left(0, T ; H^{1}(M)\right)$ almost surely, we obtain

$$
\sum_{k=k_{0}}^{\infty}\left\|\varphi\left(2^{-k} \Delta_{g}\right) u\right\|_{L^{2}\left(J^{\left.h_{k}, H^{1}\right)}\right.}^{2} \lesssim|J| .
$$


Finally, the calculation

$$
\lim _{p \rightarrow \infty} \frac{1}{p} \sum_{k=1}^{\infty} 2^{-\frac{3 k}{p}}=\lim _{p \rightarrow \infty} \frac{1}{p}\left(\frac{1}{1-2^{-\frac{3}{p}}}-1\right)=\lim _{p \rightarrow \infty} \frac{1}{p\left(2^{\frac{3}{p}}-1\right)}=\frac{1}{3 \log (2)}
$$

yields the boundedness of the function defined by $[6, \infty) \ni p \mapsto \frac{1}{p} \sum_{k=1}^{\infty} 2^{-\frac{3 k}{p}}$ and hence,

$$
\sum_{k=1}^{\infty} 2^{-\frac{3 k}{p}} \lesssim p
$$

Using the estimates (3.17) (3.18), (3.19), and (3.20) in (3.16), we get

$$
\|u\|_{L^{2}\left(J, L^{p}\right)} \lesssim 1+(|J| p)^{\frac{1}{2}}, \quad p \in[6, \infty)
$$

which implies the assertion. The proof of Proposition 3.1 is thus completed.

We would like to continue with some remarks on seemingly natural extensions of the previous result to higher dimensions, nonlinear noise and non-compact manifolds.

Remark 3.3 We would like to comment on the case of higher dimensions $d \geq 4$. The Strichartz-endpoint is $\left(2, \frac{2 d}{d-2}\right)$ and the use of Lemma 2.5 leads to the restriction $\alpha \leq 1+\frac{2}{d-2}$. The corresponding estimate in (3.16) has to be replaced by

$$
\begin{aligned}
\|u\|_{L^{2}\left(J, L^{p}\right)} \lesssim & \left\|\psi\left(\Delta_{g}\right) u\right\|_{L^{2}\left(J, L^{p}\right)}+\sum_{k=1}^{k_{0}-1}\left\|\varphi\left(2^{-k} \Delta_{g}\right) u\right\|_{L^{2}\left(J, L^{p}\right)} \\
& +\sum_{k=k_{0}}^{\infty} 2^{-\frac{k}{2}\left(\frac{d}{p}-v(d)\right)}\left\|\varphi\left(2^{-k} \Delta_{g}\right) u\right\|_{L^{2}\left(J, H^{1}\right)} \\
& +\sum_{k=k_{0}}^{\infty}\left[2^{-\frac{k}{2}\left(\frac{d}{p}-v(d)+\frac{1}{2}\right)}+2^{-\frac{k}{2}\left(\frac{d}{p}-v(d)+1\right)}+2^{-\frac{k}{2}\left(\frac{d}{p}-v(d)+\frac{1}{2}\right)}\right]
\end{aligned}
$$

for $p \geq \frac{2 d}{d-2}$, where we set $v(d):=\frac{d-3}{2}$. Hence, the convergence of the sums requires an upper bound on $\mathrm{p}$, which destroys the uniqueness proof below such that the case $d \geq 4$ remains an open problem. In fact, this problem occurs since the scaling condition for the Strichartz exponents, Sobolev embeddings and Bernstein inequalities are more restrictive in higher dimensions and therefore, the restriction to $d=3$ is of deterministic nature.

Remark 3.4 In the proof of Proposition 3.1, we did not need the optimal estimates for the correction term $\mu$ and the stochastic integral. In fact, it is possible to generalize the argument and show the estimate

$$
\|u\|_{L^{2}\left(J, L^{p}\right)} \lesssim 1+(|J| p)^{\frac{1}{2}} \quad \text { a.s., } \quad p \in[6, \infty),
$$


for martingale solutions of the equation

$$
\left\{\begin{aligned}
\mathrm{d} u(t) & =\left(\mathrm{i} \Delta_{g} u(t)-\mathrm{i} \lambda|u(t)|^{\alpha-1} u(t)+\mu\left(|u(t)|^{2(\gamma-1)} u(t)\right)\right) \mathrm{d} t \\
& -\mathrm{i} B\left(|u(t)|^{\gamma-1} u(t)\right) \mathrm{d} W(t), \\
u(0) & =u_{0},
\end{aligned}\right.
$$

with nonlinear noise of power $\gamma \in[1,2)$. However, we do not know if this equation has a solution, since the existence theory developed in [9] only applies for $\gamma=1$. Moreover, it is unclear how to apply these estimates in order to prove the pathwise uniqueness since the arguments below rely on the linearity of the noise. Hence, the case of Eq. (3.21) remains another open problem.

Remark 3.5 Let us comment on the case of possibly non-compact manifolds with bounded geometry. In the two dimensional setting, the Strichartz estimates from [16] with an additional loss of $\varepsilon$ regularity were sufficient to prove uniqueness, see [9], Section 7. In fact, these estimates correspond to the localized Strichartz estimates of the form

$$
\left\|t \mapsto e^{\mathrm{i} t \Delta_{g}} \psi_{m, \frac{1}{2}}\left(-h^{2} \Delta_{g}\right) x\right\|_{L^{q}\left(J, L^{p}\right)} \leq C_{\varepsilon}\|x\|_{L^{2}}, \quad|J| \leq \beta_{\varepsilon} h^{1+\varepsilon},
$$

for all $\varepsilon>0$ and some $C_{\varepsilon}>0$ and $\beta_{\varepsilon}>0$, where we denote $\psi_{m, a}(\lambda):=\lambda^{m} e^{-a \lambda}$ for $m \in \mathbb{N}$ and $a>0$. A continuous version of the Littlewood-Paley inequality which can substitute (2.10) is given by

$\|f\|_{L^{p}} \approx\left\|\varphi_{m, a}\left(-\Delta_{g}\right) f\right\|_{L^{p}}+\left\|\left(\int_{0}^{1}\left|\psi_{m, a}\left(-h^{2} \Delta_{g}\right) f\right|^{2} \frac{\mathrm{d} h}{h}\right)^{\frac{1}{2}}\right\|_{L^{p}}, \quad f \in L^{p}(M)$,

for $\varphi_{m, a}(\lambda):=\int_{\lambda}^{\infty} \psi_{m, a}(t) \frac{\mathrm{d} t}{t}$, see [16], Theorem 2.8. Based on (3.22) and (3.23), we can argue similarly as in the proof of Proposition 3.1 and end up with the estimate

$$
\|u\|_{L^{2}\left(J, L^{p}\right)} \lesssim 1+\mid J^{\frac{1}{2}}\left(\frac{p}{6-\varepsilon p}\right)^{\frac{1}{2}} \quad \text { a.s. }
$$

for each $\varepsilon>0$ and $p \in\left[6,6 \varepsilon^{-1}\right.$ ) with an implicit constant which goes to infinity for $\varepsilon \rightarrow 0$. The upper bound on $p$ is due to the fact that the additional $\varepsilon$ in (3.22) weakens the estimates of the critical term containing the derivative $\chi_{j}^{\prime}$ of the temporal cut-off and enlarges the number of summands in (3.14). As in the case of higher dimensions than $d=3$, the uniqueness argument breaks down since a limit process $p \rightarrow \infty$ is no longer possible.

So far, we only used the topological properties of the noise, i.e.

$$
B \in \mathcal{L}\left(H^{1}(M), \operatorname{HS}\left(Y, H^{1}(M)\right)\right), \quad \mu \in \mathcal{L}\left(H^{1}(M)\right) .
$$


Now, the Stratonovich structure and the symmetry of the operators $B_{m}$ for $m \in \mathbb{N}$ come into play to prove the following representation formula for the $L^{2}$-distance of two solutions.

Lemma 3.6 Let $d=3$ and $\alpha \in(1,3]$. Let $\left(\Omega, \mathcal{F}, \mathbb{P}, W, \mathbb{F}, u_{j}\right), j=1,2$, be solutions of Problem (1.1). Then, we have

$$
\begin{aligned}
& \left\|u_{1}(t)-u_{2}(t)\right\|_{L^{2}}^{2} \\
& =2 \int_{0}^{t} \operatorname{Re}\left(u_{1}(s)-u_{2}(s),-\mathrm{i} \lambda\left|u_{1}(s)\right|^{\alpha-1} u_{1}(s)+\mathrm{i} \lambda\left|u_{2}(s)\right|^{\alpha-1} u_{2}(s)\right)_{L^{2}} \mathrm{~d} s
\end{aligned}
$$

almost surely for all $t \in[0, T]$.

Note that the RHS of (3.24) only contains the terms induced by the nonlinearity. In particular, the stochastic integral vanishes, which will enable us to use the pathwise estimate from Proposition 3.1 to prove uniqueness.

Proof We restrict ourselves to a formal argumentation. Similarly to [9], Proposition 6.5 , our reasoning can be rigorously justified by a regularization procedure based on Yosida approximations $R_{\lambda}:=\lambda\left(\lambda-\Delta_{g}\right)^{-1}$ for $\lambda>0$. The function $\mathcal{M}: L^{2}(M) \rightarrow$ $\mathbb{R}$ defined by $\mathcal{M}(v):=\|v\|_{L^{2}}^{2}$ is twice continuously Fréchet-differentiable with

$$
\mathcal{M}^{\prime}[v] h_{1}=2 \operatorname{Re}\left(v, h_{1}\right)_{L^{2}}, \mathcal{M}^{\prime \prime}[v]\left[h_{1}, h_{2}\right]=2 \operatorname{Re}\left(h_{1}, h_{2}\right)_{L^{2}}
$$

for $v, h_{1}, h_{2} \in L^{2}(M)$. We set $w:=u_{1}-u_{2}$. Then, a formal application of the Itô formula yields

$$
\begin{aligned}
\|w(t)\|_{L^{2}}^{2}= & 2 \int_{0}^{t} \operatorname{Re}\left(w(s), \mathrm{i} \Delta_{g} w(s)-\mathrm{i}\left|u_{1}(s)\right|^{\alpha-1} u_{1}(s)+\mathrm{i}\left|u_{2}(s)\right|^{\alpha-1} u_{2}(s)\right)_{L^{2}} \mathrm{~d} s \\
& +2 \int_{0}^{t} \operatorname{Re}(w(s), \mu(w(s)))_{L^{2}} \mathrm{~d} s-2 \int_{0}^{t} \operatorname{Re}(w(s), \mathrm{i} B w(s) \mathrm{d} W(s))_{L^{2}} \\
& +\sum_{m=1}^{\infty} \int_{0}^{t}\left\|B_{m} w(s)\right\|_{L^{2}}^{2} \mathrm{~d} s
\end{aligned}
$$

almost surely for all $t \in[0, T]$. Since $\Delta_{g}$ is selfadjoint, we get $\operatorname{Re}\left(w, \mathrm{i} \Delta_{g} w\right)_{L^{2}}=0$. From the symmetry of $B_{m}, m \in \mathbb{N}$, we infer $\operatorname{Re}\left(w, \mathrm{i} B_{m} w\right)_{L^{2}}=0$ and thus, we obtain

$$
\int_{0}^{t} \operatorname{Re}(w(s), \mathrm{i} B w(s) \mathrm{d} W(s))_{L^{2}}=0 .
$$

Moreover, we simplify

$$
2 \operatorname{Re}(w(s), \mu(w(s)))_{L^{2}}=-\sum_{m=1}^{\infty} \operatorname{Re}\left(w(s), B_{m}^{2} w(s)\right)_{L^{2}}=-\sum_{m=1}^{\infty}\left\|B_{m} w(s)\right\|_{L^{2}}^{2} .
$$


Therefore, we have

$$
\|w(t)\|_{L^{2}}^{2}=2 \int_{0}^{t} \operatorname{Re}\left(w(s),-\mathrm{i}\left|u_{1}(s)\right|^{\alpha-1} u_{1}(s)+\mathrm{i}\left|u_{2}(s)\right|^{\alpha-1} u_{2}(s)\right)_{L^{2}} \mathrm{~d} s
$$

almost surely for all $t \in[0, T]$.

We close with the proof of our main Theorem 1.1. We prove the uniqueness by applying a strategy developed by Yudovich, [49], for the Euler equation. In the context of the NLS, it was first used by Vladimirov in [48], Ogawa and Ozawa in [40] and [42]. They looked at $2 D$ domains and used the Trudinger type inequalities to control the growth of the $L^{p}$-norms for $p \rightarrow \infty$. A generalization of this argument to the stochastic case in $2 D$ is straightforward and can be found in [29], Subsection 5.2. Following Burq, Gérard and Tzvetkov in the case without boundary, the Yudovich-strategy in combination with the Strichartz estimates as an improvement of the Trudinger inequality was also applied it to the deterministic NLS on compact $3 D$ manifolds with boundary by Blair, Smith and Sogge in [17].

Proof of Theorem 1.1 Step 1 Let us take two solutions $u_{1}, u_{2} \in L^{2}\left(\Omega, L^{\infty}(0, T\right.$; $\left.H^{1}(M)\right)$ ). Using Proposition 3.1, we choose a null set $N_{1} \in \mathcal{F}$ with

$$
\left\|u_{j}(\cdot, \omega)\right\|_{L^{2}\left(J, L^{p}\right)} \lesssim \omega 1+(|J| p)^{\frac{1}{2}}, \quad \omega \in \Omega \backslash N_{1},
$$

for each interval $J \subset[0, T]$ and $p \geq 6$. By Corollary 3.6, we choose a null set $N_{2} \in F$ such that

$$
\begin{aligned}
& \left\|u_{1}(t)-u_{2}(t)\right\|_{L^{2}}^{2} \\
& =2 \int_{0}^{t} \operatorname{Re}\left(u_{1}(s)-u_{2}(s),-\mathrm{i} \lambda\left|u_{1}(s)\right|^{\alpha-1} u_{1}(s)+\mathrm{i} \lambda\left|u_{2}(s)\right|^{\alpha-1} u_{2}(s)\right)_{L^{2}} \mathrm{~d} s
\end{aligned}
$$

holds on $\Omega \backslash N_{2}$ for all $t \in[0, T]$. In particular, this leads to the weak differentiability of the map $G:=\left\|u_{1}-u_{2}\right\|_{L^{2}}^{2}$ on $\Omega \backslash N_{2}$ and to the estimate

$$
\begin{aligned}
\left|G^{\prime}(t)\right| & =\left|2 \operatorname{Re}\left(u_{1}(s)-u_{2}(s),-\mathrm{i} \lambda\left|u_{1}(s)\right|^{\alpha-1} u_{1}(s)+\mathrm{i} \lambda\left|u_{2}(s)\right|^{\alpha-1} u_{2}(s)\right)_{L^{2}}\right| \\
& \lesssim \int_{M}\left|u_{1}(s, x)-u_{2}(s, x)\right|^{2}\left(\left|u_{1}(s, x)\right|^{\alpha-1}+\left|u_{2}(s, x)\right|^{\alpha-1}\right) \mathrm{d} x .
\end{aligned}
$$

The Sobolev embedding $H^{1}(M) \hookrightarrow L^{6}(M)$ yields $u_{j} \in L^{\infty}\left(0, T ; L^{6}(M)\right), j=1,2$, almost surely. Moreover, we have the mild representation

$$
\begin{aligned}
\mathrm{i} u_{j}(t)= & \mathrm{i} e^{\mathrm{i} t \Delta_{g}} u_{0}+\int_{0}^{t} e^{\mathrm{i}(t-\tau) \Delta_{g}} \lambda\left|u_{j}(\tau)\right|^{\alpha-1} u_{j}(\tau) \mathrm{d} \tau+\mathrm{i} \int_{0}^{t} e^{\mathrm{i}(t-\tau) \Delta_{g}} \mu\left(u_{j}(\tau)\right) \mathrm{d} \tau \\
& +\int_{0}^{t} e^{\mathrm{i}(t-\tau) \Delta_{g}} \mathrm{~B}\left(u_{j}(\tau)\right) \mathrm{d} W(\tau)
\end{aligned}
$$


almost surely for all $t \in[0, T]$ in $H^{-1}(M)$ for $j=1,2$. As a consequence of $\alpha \in(1,3]$ and $u_{j} \in L^{\infty}\left(0, T ; L^{6}(M)\right)$, each of the terms on the RHS is in $L^{2}(M)$. In particular, we obtain $u_{j} \in C\left([0, T], L^{2}(M)\right), j=1,2$, almost surely and thus, we can take another null set $N_{3} \in F$ such that

$$
u_{j} \in L^{\infty}\left(0, T ; L^{6}(M)\right) \cap C\left([0, T], L^{2}(M)\right) \text { on } \Omega \backslash N_{3} .
$$

Now, we define $\Omega_{1}:=\Omega \backslash\left(N_{1} \cup N_{2} \cup N_{3}\right)$ and fix $\omega \in \Omega_{1}$. We take a sequence $\left(p_{n}\right)_{n \in \mathbb{N}} \in[6, \infty)^{\mathbb{N}}$ with $p_{n} \rightarrow \infty$ as $n \rightarrow \infty$. We fix $n \in \mathbb{N}$ and define $q_{n}:=\frac{p_{n}}{\alpha-1}$. By the estimate (3.28) and the Hölder inequality with exponents $\frac{1}{q_{n}^{\prime}}+\frac{1}{q_{n}}=1$, we get

$$
\left|G^{\prime}(t)\right| \lesssim\left\|u_{1}(t)-u_{2}(t)\right\|_{L^{2 q_{n}^{\prime}}}^{2}\left\|\left|u_{1}(t)\right|^{\alpha-1}+\left|u_{2}(t)\right|^{\alpha-1}\right\|_{L^{q_{n}}}, \quad t \in[0, T] .
$$

The choice of $q_{n}$ yields $2 q_{n}^{\prime} \in[2,6]$ and for $\theta:=\frac{3}{2 q_{n}} \in(0,1)$, we have $\frac{1}{2 q_{n}^{\prime}}=\frac{1-\theta}{2}+\frac{\theta}{6}$. Hence, we obtain

$$
\begin{aligned}
& \left\|u_{1}(t)-u_{2}(t)\right\|_{L^{2 q_{n}^{\prime}}}^{2} \leq\left\|u_{1}(t)-u_{2}(t)\right\|_{L^{2}}^{2-\frac{3}{q_{n}}}\left\|u_{1}(t)-u_{2}(t)\right\|_{L^{6}}^{\frac{3}{q_{n}}} \\
& \leq\left\|u_{1}(t)-u_{2}(t)\right\|_{L^{2}}^{2-\frac{3}{q_{n}}}\left\|u_{1}-u_{2}\right\|_{L^{\infty}\left(0, T ; L^{6}\right)}^{\frac{3}{q_{n}}}
\end{aligned}
$$

by interpolation. We choose a constant $C_{1}>0$ such that

$$
\left\|u_{1}\right\|_{L^{\infty}\left(0, T ; L^{6}\right)}+\left\|u_{2}\right\|_{L^{\infty}\left(0, T ; L^{6}\right)} \leq C_{1}
$$

which leads to

$$
\left|G^{\prime}(t)\right| \lesssim C_{1}^{\frac{3}{q_{n}}} G(t)^{1-\frac{3}{2 q_{n}}}\left[\left\|u_{1}(t)\right\|_{L^{p_{n}}}^{\alpha-1}+\left\|u_{2}(t)\right\|_{L^{p_{n}}}^{\alpha-1}\right]
$$

Step 2 We argue by contradiction and assume that there is $t_{2} \in[0, T]$ with $G\left(t_{2}\right)>$ 0 . By the continuity of $G$, we get

$$
\exists t_{1} \in\left[0, t_{2}\right): G\left(t_{1}\right)=0 \text { and } \forall t \in\left(t_{1}, t_{2}\right): G(t)>0 \text {. }
$$

We set $J_{\varepsilon}:=\left(t_{1}, t_{1}+\varepsilon\right)$ with $\varepsilon \in\left(0, t_{2}-t_{1}\right)$ to be chosen later. By the weak chain rule, see [28, Theorem 7.8] and (3.29), we get

$$
\begin{aligned}
G(t)^{\frac{3}{2 q_{n}}} & =\frac{3}{2 q_{n}} \int_{t_{1}}^{t} G^{\prime}(s) G(s)^{\frac{3}{2 q_{n}}-1} \mathrm{~d} s \\
& \lesssim \frac{3}{2 q_{n}} C_{1}^{\frac{3}{q_{n}}} \int_{t_{1}}^{t}\left[\left\|u_{1}(s)\right\|_{L^{p_{n}}}^{\alpha-1}+\left\|u_{2}(s)\right\|_{L^{p n}}^{\alpha-1}\right] \mathrm{d} s, \quad t \in J_{\varepsilon} .
\end{aligned}
$$


By another application of the the Hölder inequality with exponents $\frac{2}{\alpha-1}$ and $\frac{2}{3-\alpha}$, we infer that

$$
G(t)^{\frac{3}{2 q_{n}}} \lesssim \frac{3}{2 q_{n}} C_{1}^{\frac{3}{q_{n}}}\left[\left\|u_{1}\right\|_{L^{2}\left(t_{1}, t ; L^{p_{n}}\right)}^{\alpha-1}+\left\|u_{2}\right\|_{L^{2}\left(t_{1}, t ; L^{p_{n}}\right)}^{\alpha-1}\right] \varepsilon^{\frac{3-\alpha}{2}}, \quad t \in J_{\varepsilon} .
$$

Now, we are in the position to apply (3.26) and we obtain

$$
G(t)^{\frac{3}{2 q_{n}}} \lesssim \frac{3}{2 q_{n}} C_{1}^{\frac{3}{q_{n}}}\left(1+\left(\varepsilon p_{n}\right)^{\frac{\alpha-1}{2}}\right) \varepsilon^{\frac{3-\alpha}{2}}, \quad t \in J_{\varepsilon} .
$$

In particular, there is a constant $C>0$ such that for all $t \in J_{\varepsilon}$ it holds that

$$
\begin{aligned}
G(t) & \leq C_{1}^{2}\left(\frac{3 C}{2 q_{n}}\left(1+\left(\varepsilon(\alpha-1) q_{n}\right)^{\frac{\alpha-1}{2}}\right) \varepsilon^{\frac{3-\alpha}{2}}\right)^{\frac{2 q_{n}}{3}} \\
& \leq C_{1}^{2}\left(\frac{3 C}{2 q_{n}}\left(1+\varepsilon^{\frac{\alpha-1}{2}}(\alpha-1) q_{n}\right) \varepsilon^{\frac{3-\alpha}{2}}\right)^{\frac{2 q_{n}}{3}}=: b_{n},
\end{aligned}
$$

where we used $p_{n}:=q_{n}(\alpha-1)$ and $\frac{\alpha-1}{2} \in(0,1]$.

Step 3 We aim to show that the sequence $\left(b_{n}\right)_{n \in \mathbb{N}}$ on the RHS of (3.31) converges to 0 for $\varepsilon$ sufficiently small. Then, we have proved $G(t)=0$ for all $t \in J_{\varepsilon}$ which contradicts (3.30). Hence, we have $u_{1}(t)=u_{2}(t)$ almost surely for all $t \in[0, T]$.

To this end, we choose $\varepsilon \in\left(0, \min \left\{t_{2}-t_{1}, \frac{2}{3 C(\alpha-1)}\right\}\right)$. Then,

$$
\begin{aligned}
b_{n} & =C_{1}^{2}\left(\frac{3 C}{2 q_{n}}\left(1+\varepsilon^{\frac{\alpha-1}{2}}(\alpha-1) q_{n}\right) \varepsilon^{\frac{3-\alpha}{2}}\right)^{\frac{2 q_{n}}{3}} \\
& =C_{1}^{2}\left(\frac{3 C \varepsilon(\alpha-1)}{2}\right)^{\frac{2 q_{n}}{3}}\left(\frac{1}{\varepsilon^{\frac{\alpha-1}{2}}(\alpha-1) q_{n}}+1\right)^{\frac{2 q_{n}}{3}} \stackrel{n \rightarrow \infty}{\longrightarrow} 0 .
\end{aligned}
$$

The proof of Theorem 1.1 is thus completed.

\section{Concluding remarks and open questions}

To the best of our understanding there exists in the literature at least three different methods of studying the question of the existence of solutions to stochastic Nonlinear Schrödinger Equation (NLS), i.e.

(i) The compactness method,

(ii) The Banach Fixed Point Theorem or the Picard iteration scheme,

(iii) The Doss-Sussmann transformation.

In our recent joint paper [9] we proved the existence of solutions to the stochastic NLS by employing the compactness method. The method of proving the existence of solutions based on the Banach Fixed Point Theorem or the Picard iteration scheme is the 
original one, see the celebrated papers [21] and [22]) by de Bouard and Debussche, as well as more recent papers [10] and [30], and references therein. One important unifying feature of these two approaches is the use of the deterministic [7] and the stochastic Strichartz estimates. The third approach goes back to papers [23] by Doss and [45] by Sussman for stochastic ordinary equations. This approach, usually called the DossSussmann method, has been used with the so-called bilinear noise in the case of stochastic parabolic equations by Acquistapace and Terreni [1] and by the first named authour et al in [2]. A generalisation of that approach based on the Kunita's stochastic characteristics has also been used by a multiple of authors, e.g. the first named author and Flandoli [3], Lisei [39], Röger and Weber in [43], Chugreeva+Melcher [19] and references therein. This generalisation has also been discussed as an alternative proof by the first named authour et al in [5] for stochastic Euler Equations. The third method has also been employed for the stochastic NLS by Barbu, Röckner and Zhang in [14], see also [50], where it was called a "rescaling" method, to prove in particular the existence and the uniqueness of solutions. As in the earlier cited papers these papers treat the so called bilinear noise and have been generalised, to allow in particular non-linear noise coefficients, using the second method by the second named author in [30]. Let us point out that in our previous paper [9] we also studied stochastic NLS driven by bilinear noise but some generalisations to non-linear noise coefficients have been found in the $\mathrm{PhD}$ thesis [29] by the second named author. One fundamental difference between the first and the second methods on the one hand and the Doss-Sussman method on the other is that the latter only works for bilinear noise while the former for more general noise coefficients. Nevertheless, a natural question emerges whether the results from our previous paper [9] can be fully proven by using the Doss-Sussman method? Since the present paper essentially works only for bilinear noise, a second natural question is whether it is possible to prove our uniqueness result from the present article by employing the Doss-Sussman method? The difficulty one would encounter in undertaking such a challenge is that after applying the Doss-Sussman transformation, the NLS in the Itô-Stratonovitch form becomes a family of deterministic equations but with the Laplace-Beltrami operator being replaced by a time-dependent second-order operator containing first-order terms and we simply do not know how to treat such equations. Even classical Strichartz estimates are problematic. We find these an interesting questions and hope that some other researchers will find them interesting as well.

We finish these comments with posing another interesting open problem: Is it possible to generalize the uniqueness to other geometries (in particular bounded or unbounded do- mains and non-compact manifolds). The existence in the latter cases is proved in the recent paper [31] by the second named author. In Remark 3.5 we explained why we couldn't apply the approach from the present paper to prove uniqueness for these geometries.

Finally we would like to discuss our Corollary 1.2 about the existence of the strong solutions. Our approach of proving this result consists of three steps. The first step is to prove the existence of weak/martingale solutions. This step has been successfully concluded in our previous paper [9]. The second step is to prove the pathwise uniqueness of weak/martingale solutions. This step has been successfully concluded in our current paper. The third step is to apply the infinite dimensional Yamada-Watanabe 
Theorem. All these three steps have been implemented for stochastic reaction diffusion equations in a rather classical paper by the first named author and Gatarek in [6], where the classical version Yamada-Watanabe Theorem from [32] has been used. A proper formulation and a full detailed proof of the infinite dimensional Yamada-Watanabe Theorem has been first presented by Ondreját in [41] for mild solutions and by Kunze [36] for weak solutions. Here we use the latter as it fits better our framework. However, since the existence of solutions is obtained by a compactness argument, it is probably possible to use the Gyongy and Krylov Lemma, see [26, Lemma 1] to prove that in fact the approximations converge in probability. As far as we understand, this approach has been recently used by Crisan, Flandoli and Holm [18] and it still required the use of the Skorokhod embedding theorem. It would of interest to see if this approach works for the class of stochastic NLS studied in the present paper. One possible source of difficulties could be that the original result by Gyongy-Krylov works in the framework of Polish spaces while the existence proof from our first paper [9] a certain class of non-Polish spaces as the Jakubowski-Skorokhod Theorem by Jakubowski from [33]. But, it happens that the Gyongy-Krylov Lemma has been recently generalised to the same framework as used in [33] in a recent monograph by Breit, Hofmanova and Feireisl [4].

Acknowledgements We gratefully acknowledge financial support by the Deutsche Forschungsgemeinschaft (DFG) through CRC 1173. We would like to thank Arnaud Debussche for useful questions and comments leading to our last section.

Data Availability Data sharing not applicable to this article as no datasets were generated or analysed during the current study.

Open Access This article is licensed under a Creative Commons Attribution 4.0 International License, which permits use, sharing, adaptation, distribution and reproduction in any medium or format, as long as you give appropriate credit to the original author(s) and the source, provide a link to the Creative Commons licence, and indicate if changes were made. The images or other third party material in this article are included in the article's Creative Commons licence, unless indicated otherwise in a credit line to the material. If material is not included in the article's Creative Commons licence and your intended use is not permitted by statutory regulation or exceeds the permitted use, you will need to obtain permission directly from the copyright holder. To view a copy of this licence, visit http://creativecommons.org/licenses/by/4.0/.

\section{References}

1. Acquistapace, P., Terreni, B.: An approach to Ito linear equations in Hilbert spaces by approximation of white noise with coloured noise. Stoch. Anal. Appl. 2(2), 131-186 (1984)

2. Brzeźniak, Z., Capiński, M., Flandoli, F.: A convergence result for stochastic partial differential equations. Stochastics 24(4), 423-445 (1988)

3. Brzeźniak, Z., Flandoli, F.: Almost sure approximation of Wong-Zakai type for stochastic partial differential equations. Stoch. Process. Appl. 55(2), 329-358 (1995)

4. Breit, D., Feireisl, E., Hofmanová, M.: Stochastically Forced Compressible Fluid Flows. De Gruyter Series in Applied and Numerical Mathematics. De Gruyter, Berlin (2018)

5. Brzeźniak, Z., Flandoli, F., Maurelli, M.: Existence and uniqueness for stochastic 2D Euler flows with bounded vorticity. Arch. Ration. Mech. Anal. 221(1), 107-142 (2016)

6. Brzeźniak, Z., Ga̧tarek, D.: Martingale solutions and invariant measures for stochastic evolution equations in Banach spaces. Stoch. Process. Appl. 84(2), 187-225 (1999)

7. Burq, N., Gérard, P., Tzvetkov, N.: Strichartz inequalities and the nonlinear Schrödinger equation on compact manifolds. Am. J. Math. 126(3), 569-605 (2004) 
8. Brzeźniak, Z., Hornung, F., Manna, U.: Weak martingale solutions for the stochastic nonlinear schrödinger equation driven by pure jump noise. Stoch. Partial Differ. Equ. Anal. Comput. 8(1), 1-53 (2020)

9. Brzeźniak, Z., Hornung, F., Weis, L.: Martingale solutions for the stochastic nonlinear Schrödinger equation in the energy space. Probab. Theory Relat. Fields 174(3-4), 1273-1338 (2019)

10. Brzeźniak, Z., Millet, A.: On the stochastic Strichartz estimates and the stochastic nonlinear Schrödinger equation on a compact Riemannian manifold. Potential Anal. 41(2), 269-315 (2014)

11. Bolleyer, A.: Spectrally Localized Strichartz Estimates and Nonlinear Schrödinger Equations. PhD thesis, Karlsruhe Institute of Technology (2015)

12. Bouclet, J.-M.: Littlewood-Paley decompositions on manifolds with ends. Bull. Soc. Math. France 138(1), 1-37 (2010)

13. Brzeźniak, Z.: On stochastic convolution in Banach spaces and applications. Stoch. Int J Prob Stoch Process. 61(3-4), 245-295 (1997)

14. Barbu, V., Röckner, M., Zhang, D.: Stochastic nonlinear Schrödinger equations with linear multiplicative noise: rescaling approach. J. Nonlinear Sci. 24(3), 383-409 (2014)

15. Barbu, V., Röckner, M., Zhang, D.: Stochastic nonlinear Schrödinger equations. Nonlinear Anal. Theory Methods Appl. 136, 168-194 (2016)

16. Bernicot, F., Samoyeau, V.: Dispersive estimates with loss of derivatives via the heat semigroup and the wave operator. Annali della scuola normale superiore di pisa. Cl. Sci. 17(3), 969-1029 (2017)

17. Blair, M.D., Smith, H.F., Sogge, C.D.: Strichartz estimates and the nonlinear Schrödinger equation on manifolds with boundary. Math. Ann. 354(4), 1397-1430 (2012)

18. Crisan, D., Flandoli, F., Holm, D.: Solution properties of a 3D stochastic Euler fluid equation. J. Nonlinear Sci. 29(3), 813-870 (2019)

19. Chugreeva, O., Melcher, C.: Vortices in a stochastic parabolic Ginzburg-Landau equation. Stoch. Partial Differ. Equ. Anal. Comput. 5(1), 113-143 (2017)

20. Cheung, K., Mosincat, R.: Stochastic nonlinear Schrödinger equations on tori. Stoch. Partial Differ. Equ. Anal. Comput. 7(2), 169-208 (2019)

21. de Bouard, A., Debussche, A.: A stochastic nonlinear Schrödinger equation with multiplicative noise. Commun. Math. Phys. 205(1), 161-181 (1999)

22. de Bouard, A., Debussche, A.: The stochastic nonlinear Schrödinger equation in $H^{1}$. Stoch. Anal. Appl. 21(1), 97-126 (2003)

23. Doss, H.: Liens entre équations différentielles stochastiques et ordinaires. Ann. Inst. H. Poincaré Sect. B (N.S.) 13(2), 99-125 (1977)

24. Duong, X.T., Ouhabaz, E.M., Sikora, A.: Plancherel-type estimates and sharp spectral multipliers. J. Funct. Anal. 196(2), 443-485 (2002)

25. Da Prato, G., Zabczyk, P.J.: Stochastic Equations in Infinite Dimensions. Cambridge University Press, Cambridge (2014)

26. Gyöngy, I., Krylov, N.: Existence of strong solutions for Itô's stochastic equations via approximations. Probab. Theory Relat. Fields 105(2), 143-158 (1996)

27. Grafakos, L.: Classical Fourier Analysis, 3rd edn. Springer, New York (2014)

28. Gilbarg, D., Trudinger, N.S.: Elliptic Partial Differential Equations of Second Order. Classics in Mathematics. U.S. Government Printing Office (2001)

29. Hornung, F.: Global solutions of the nonlinear Schrödinger equation with multiplicative noise. PhD thesis, Karlsruhe Institute of Technology, (2018)

30. Hornung, F.: The nonlinear stochastic Schrödinger equation via stochastic Strichartz estimates. J. Evol. Equ. 18(3), 1085-1114 (2018)

31. Hornung, F.: The stochastic nonlinear Schrödinger equation in unbounded domains and non-compact manifolds. NoDEA Nonlinear Differ. Equ. Appl. 27, 40 (2020)

32. Ikeda, N., Watanabe, S.: Stochastic Differential Equations and Diffusion Processes. North-Holland Publishing Company, North-Holland mathematical library (1981)

33. Jakubowski, A.: The almost sure Skorokhod representation for subsequences in nonmetric spaces. Theory Probab. Appl. 42(1), 167-174 (1998)

34. Keller, D., Lisei, H.: A stochastic nonlinear Schrödinger problem in variational formulation. Nonlinear Differ. Equ. Appl. 23(2), 1-27 (2016)

35. Keel, M., Tao, T.: Endpoint Strichartz estimates. Am. J. Math. 120(5), 955-980 (1998)

36. Kunze, M.: On a class of martingale problems on Banach spaces. Electron. J. Probab. 18, 1-30 (2013) 
37. Kriegler, C., Weis, L.: Paley-Littlewood decomposition for sectorial operators and interpolation spaces. Math. Nachr. 289(11-12), 1488-1525 (2016)

38. Lablée, O.: Spectral Theory in Riemannian Geometry. EMS Textbooks in Mathematics, (2015)

39. Lisei, H.: Flows for the stochastic Navier-Stokes equation. Math. Pannon. 13(2), 223-240 (2002)

40. Ogawa, T.: A proof of Trudinger's inequality and its application to nonlinear Schrödinger equations. Nonlinear Anal. Theory Methods Appl. 14(9), 765-769 (1990)

41. Ondreját, M.: Uniqueness for stochastic evolution equations in Banach spaces. Diss. Math. (Rozprawy Mat.) 426, 63 (2004)

42. Ogawa, T., Ozawa, T.: Trudinger type inequalities and uniqueness of weak solutions for the nonlinear Schrödinger mixed problem. J. Math. Anal. Appl. 155(2), 531-540 (1991)

43. Röger, M., Weber, H.: Tightness for a stochastic Allen-Cahn equation. Stoch. Partial Differ. Equ. Anal. Comput. 1(1), 175-203 (2013)

44. Strichartz, R.S.: Analysis of the Laplacian on the complete Riemannian manifold. J. Funct. Anal. 52(1), 48-79 (1983)

45. Sussmann, H.J.: On the gap between deterministic and stochastic ordinary differential equations. Ann. Probab. 6(1), 19-41 (1978)

46. Triebel, H.: Theory of Function Spaces II. Monographs in Mathematics; 84. Birkhäuser, Basel [u.a.], (1992)

47. M: Uhl.: Spectral Multiplier Theorems of Hörmander Type via Generalized Gaussian Estimates. PhD thesis, Karlsruhe Institute of Technology, (2011)

48. Vladimirov, M.V.: Solvability of a mixed problem for the nonlinear Schrödinger equation. Math. USSR-Sbornik 58(2), 525 (1987)

49. Yudovich, V.I.: Non-stationary flows of an ideal incompressible fluid. Zhurnal Vychislitel'noi Mat. Mat. Fiziki 3(6), 1032-1066 (1963)

50. Zhang, D.: Strichartz and local smoothing estimates for stochastic dispersive equations with linear multiplicative noise. arXiv preprint arXiv:1709.03812, (2017)

Publisher's Note Springer Nature remains neutral with regard to jurisdictional claims in published maps and institutional affiliations. 